\title{
Review Paper \\ A Scoping Review of Questionnaires on Stigma of Mental Illness in Persian
}

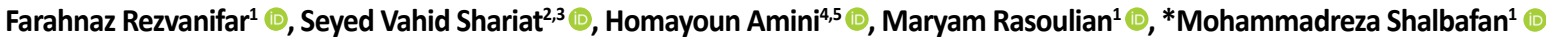

1. Mental Health Research Center, Iran University of Medical Sciences, Tehran, Iran.

2. Mental Health Research Center, Psychosocial Health Research Institute (PHRI), Iran University of Medical Sciences, Tehran, Iran.

3. School of Behavioral Sciences and Mental Health (Tehran Institute of Psychiatry), Iran University of Medical Sciences, Tehran, Iran.

4. Department of Psychiatry, Roozbeh Psychiatry Hospital, Tehran University of Medical Sciences, Tehran, Iran.

5. Psychosomatic Medicine Research Center, Tehran University of Medical Sciences, Tehran, Iran.

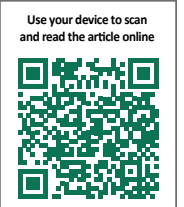

Citation Rezvanifar F, Shariat S, Amini H, Rasoulian M, Shalbafan1 M. [A Scoping Review of Questionnaires on Stigma of Mental Illness in Persian (Persian)]. Iranian Journal of Psychiatry and Clinical Psychology. 2020; 26(2):240-259. http://dx.doi. org/10.32598/ijpcp.26.2.2619.1

doi hittp://dx.doi.org/10.32598/ijpcp.26.2.2619.1

Received: 09 Sep 2019

Accepted: 12 Feb 2020 Available Online: $01 \mathrm{Jul} 2020$

Key words: Social stigma, Mental disorders, Questionnaires and surveys

\section{A B STRACT}

Objectives In order to plan according to the needs of psychiatric patients, there is a need to study different aspects of social stigma in target groups. Due to the limited access to questionnaires used for assessment of social stigma in Persian, this study aims to review all of the tools available for assessment of social stigma, which have been designed in Iran or have been translated to Persian.

Methods This is a conceptual review study. After extensive review of resources, articles related to social stigma in the health system and conducted on health care staff, students, caregivers or patients' families, psychiatric patients, mothers with children with autism, mothers with children with Down syndrome, and the general population were included in the study. We extracted the information of Persian questionnaires used in the reviewed articles. The questionnaires and additional information were collected by contacting the authors of the articles via E-mail.

Results There were 21 questionnaires for assessing social stigma in Persian language. Of these, 19 questionnaires could be accessed and analyzed. Most of the questionnaires were the translated version of other tools, and only 4 questionnaires had been developed and validated in Persian.

Conclusion Most of the questionnaires in Persian are used to measure social stigma in health care staff and students followed by caregivers or patients' families. There is limited number of questionnaires for the assessment of social stigma in general population and patients. This is in contrast to the other languages that have mainly focused on assessment of stigma in general population and patients. To date, no questionnaire in Persian exists for the assessment of social stigma in children and adolescents.

\section{Extended Abstract}

\section{Introduction}

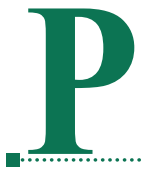

atients with mental illness generally face two major issues related to their disorder: they should deal with not only different signs and symptoms of their disorders (including anxiety, delusion, hallucination, etc.) but also with the concurrent social stigma of mental illness. The families of the patients with mental illness usually feel embarrassed and guilty about the disorder of their relative and would also experience similar social isolation and stigma.

\section{* Corresponding Author:}

Mohammadreza Shalbafan, PhD.

Address: Mental Health Research Center, Iran University of Medical Sciences, Tehran Iran.

Tel: +98 (21) 66551665

E-mail: shalbafan.mr@iums.ac.ir 
Despite the progress in the treatment of mental disorders in recent decades, the social stigma of mental illness still exists and affects the patients with mental illness and their families as well as mental health personnel. For appropriate planning for the needs of the patients with mental illness, it is necessary to study different aspects of stigma in various sub-populations.

To adequately address the need for precise assessment of the social stigma of mental illness, we need valid and reliable assessment tools. Due to the limited access to questionnaires to assess the social stigma of mental illness in Persian, we planned to review all of the assessment tools related to the social stigma of mental illness, which have been designed in Iran or translated into Persian.

\section{Methods}

We searched for both Persian and English sources. We searched the English databases, including PubMed, Google Scholar, Scopus, and PsycINFO, along with the Persian databases, including IranMedex, Scientific Information Database, Irandoc, and Magiran. We did not limit our searches for a specific period. For English databases, we combined three keywords of "Stigma" OR its related words, "Mental Health" OR its related words, and "Iran" OR the related words using the AND operator. For Persian databases we used only the Persian equivalent terms for "stigma" and no Boolean search was used.

Our search yielded 289 articles and 248 theses. Only 25 articles and 7 theses met our inclusion criteria of studying stigma, using a questionnaire in English and Persian. After removing shared questionnaires, we found 21 distinct stigma questionnaires. Then, we emailed the corresponding authors of all of the articles, and informing them about our study asked them to send us a copy of the questionnaire and surveys that they used in their study. We also asked their permission to publish the full text of their questionnaire as an appendix to our article. Finally, we gathered the whole data on 19 questionnaires.

\section{Results}

Twenty-one questionnaires and surveys were used to assess 'stigma' in the Persian language that included: Social Distance Item, Perceived Dangerousness of Mental Patients Item, The Attribution Questionnaire, The Level of Familiarity Scale, Attitudes toward Seeking Professional Psychological Help, Knowledge and Attitude about Patients with Mental Disorders and Epilepsy, Experience of Caregiving Inventory, Internalization of Stigma for Mothers of Children with Disability, Opening Minds Scale for Health Care
Providers, Perceived Stigma of Mental Illness in Nurses, Opinion about Mental Illness, Depression Stigma Scale, Stigma Scale, The Parents Internalized Stigma of Mental Illness Scale, Family Caregiver's Stigma toward Patients with Mental Illness, Opening Minds Scale for Health Care Providers, Stigma in Patients with Mental Disorders, Perceived Stigma Questionnaire, Cognitive Appraisal of Stigma-related Stress, Rejection Sensitivity Questionnaire and Perceived Stigma Scale-Revised. We could not find the Persian version of the Rejection Sensitivity Questionnaire and Perceived Stigma Scale.

\section{Discussion}

Most of the questionnaires and surveys of the social stigma of mental illness in Persian were translated version from other languages except four questionnaires that have been originally developed and validated in Persian. These four questionnaires include the Internalization of Stigma for Mothers of Children with Disability, Family Caregiver's Stigma toward Patients with Mental Illness, Perceived Stigma Questionnaire, and Perceived Stigma of Mental Illness in Nurses. Only two questionnaires assessed the stigma of mental illness within the general population (Knowledge and Attitude about Patients with Mental Disorders and Epilepsy, Opinion about Mental Illness). However, eight questionnaires are available to assess the stigma of mental illness in caregivers or family members of patients with mental disorders.

There are ten questionnaires for assessment of stigma of mental illness in health care providers, and medical and nursing students as future care providers. Few questionnaires and surveys are available in Persian for assessment of stigma in patients themselves. In the world literature, most of the questionnaires have been developed to assess stigma in the general population and then in patient. However, most of the questionnaires and surveys studied in Iran are related to care providers and students and then related to caregivers or family members of the patients. Limited questionnaires are available for assessment of stigma in general population and patients. Furthermore, we did not find any questionnaires for assessment of stigma in children and adolescents. Most of the questionnaires and surveys that are being used in Persian are translations from an English questionnaire. Interestingly, some of the questionnaires are used for a different target population than the original questionnaire without clear justifications. 


\section{Ethical Considerations}

Compliance with ethical guidelines

For all reviewed questionnaires, necessary permissions were obtained from their authors. Because of the type of article (Review), there was no need for obtaining ethical approval.

Funding

This research did not receive any grant from funding agencies in the public, commercial, or non-profit sectors.

Authors contributions

Conceptulization and design: Farahnaz Rezvanifar, Seyed Vahid Shariat, and Mohammadreza Shalbafan; Data collection: Farahnaz Rezvanifar, Mohammadreza Shalbafan, and Maryam Rasoulian; Initial draft preparation: Farahnaz Rezvanifar and Homayoun Amini; Editing \& review: All authors.

\section{Conflicts of interest}

The authors declared no conflict of interest. 


\title{
يرسش نامههاى انكَ ناشى از بيمارىهاى روانيزشكى در زبان فارسى: مطالعهاى بر اساس يك

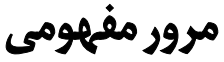

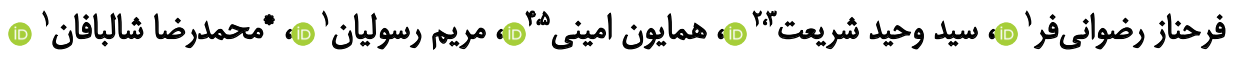 \\ ا. مركز تحقيقات بهداشت روان، دانشعاه علوميزشكى ايران، تهران، ايران.

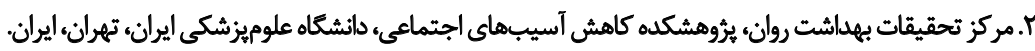

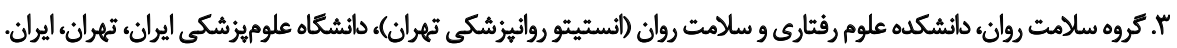

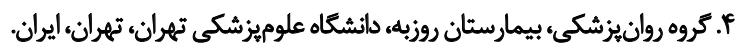

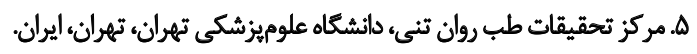

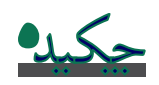

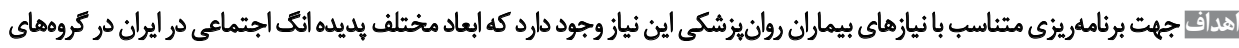

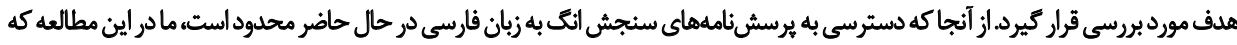

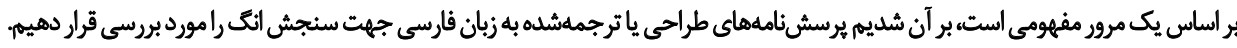

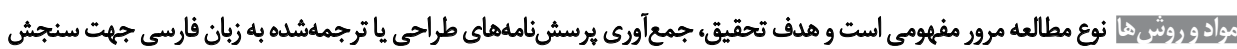

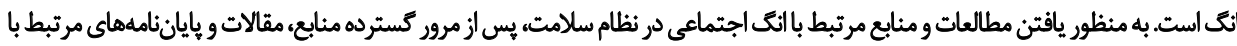

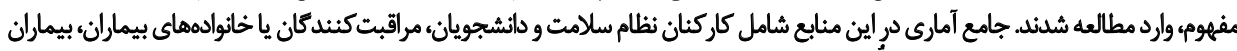

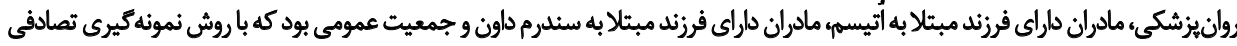

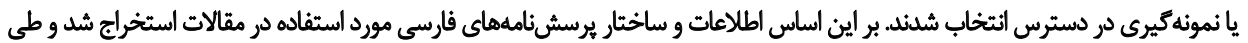

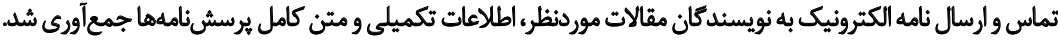

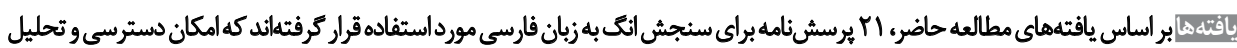

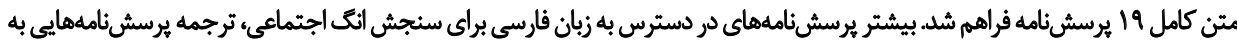

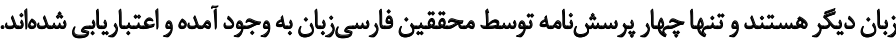

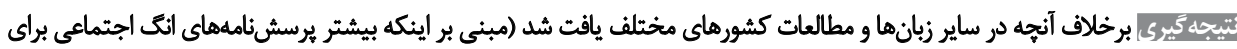

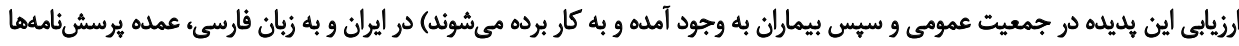

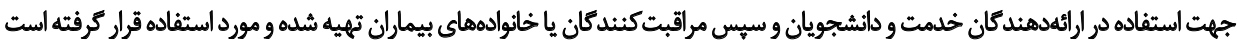

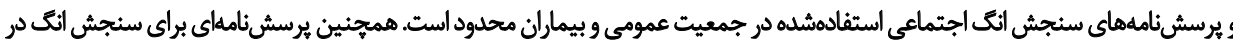

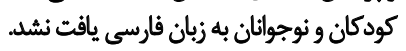

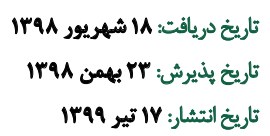

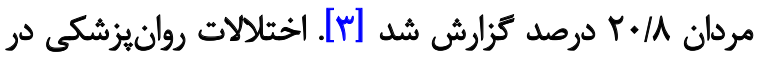

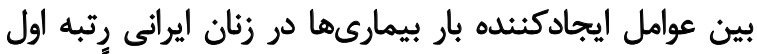

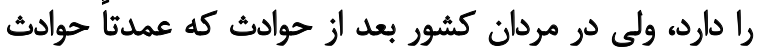

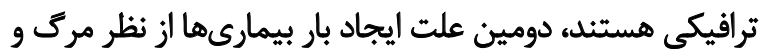

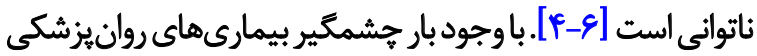

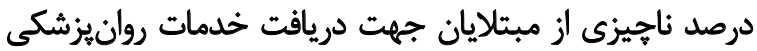

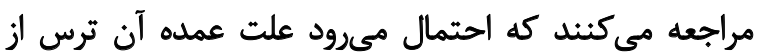

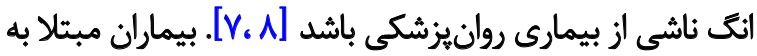

daleo

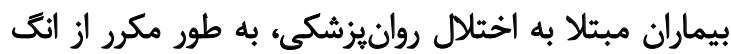

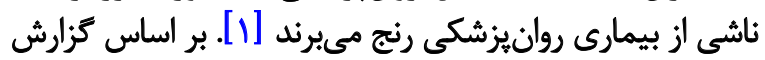

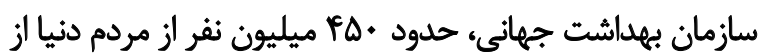

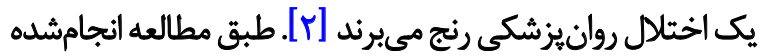

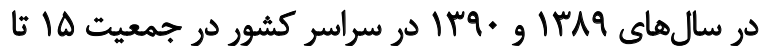

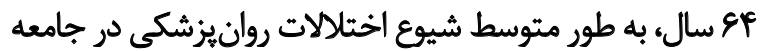

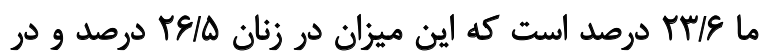

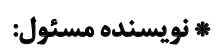
محمدرضا شالبافان

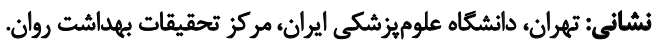
تلفئ:

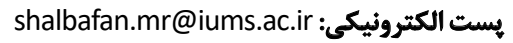


بار بيمارىهاء ناتواني درازمدت بيمارى هار را مي افزرايد و درنتيجها

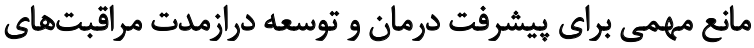

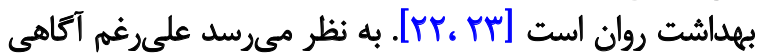

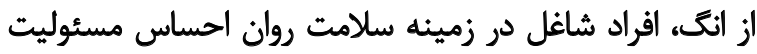

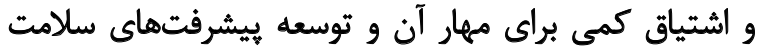

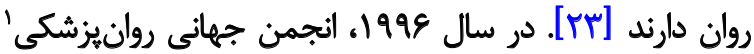

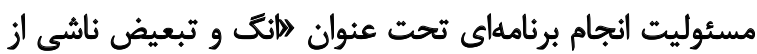

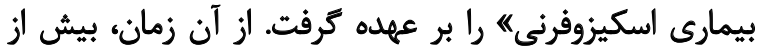

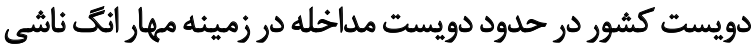

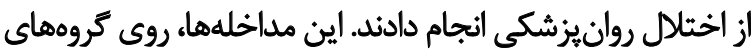

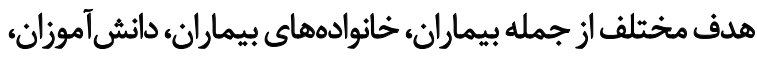

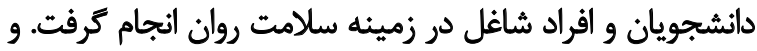

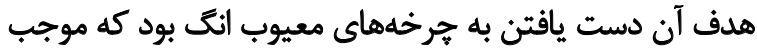

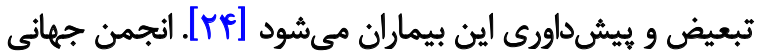

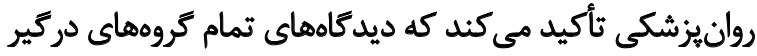

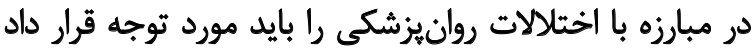

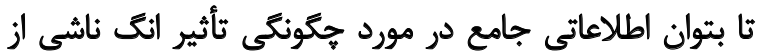

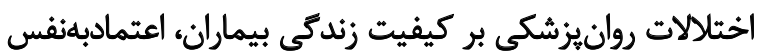

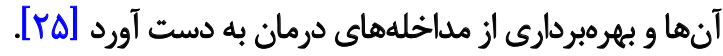

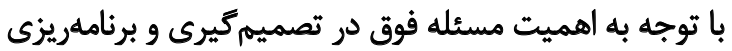

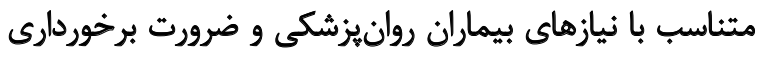

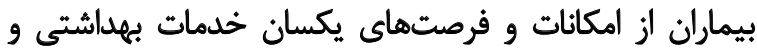

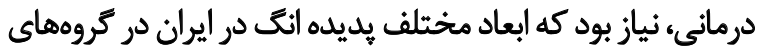

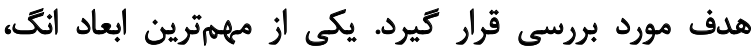

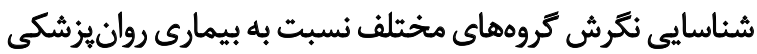

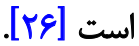

براى سنجش نكرش و بررسى انت تاكنون از شيوههاى

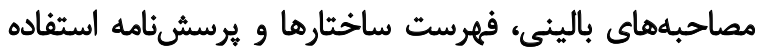

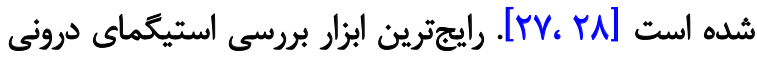

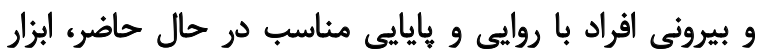

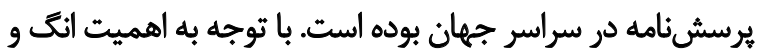

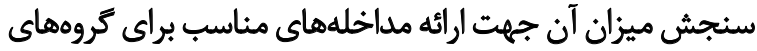

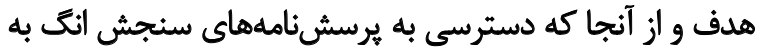

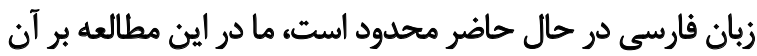

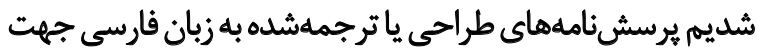

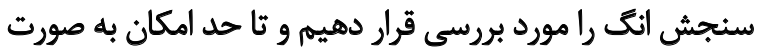

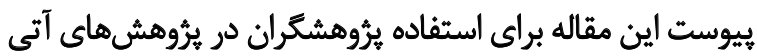
ارائه كنيه اين مقائ

ووشث

به منظور يافتن مطالعات و منابع مرتبط با انك در نظام سلامت،

1. World Psychiatric Association (WPA)
بيمارىهاى روانيزشكى اغلب با دو مسئله عمله روبهرو هستند.

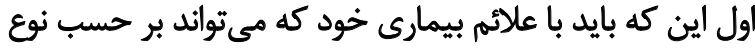

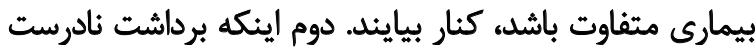

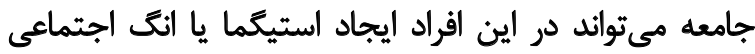

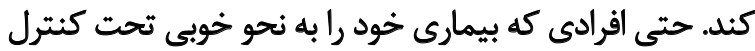

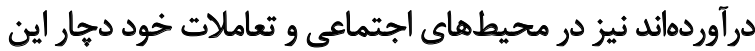

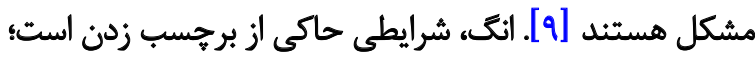

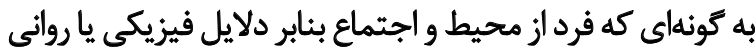

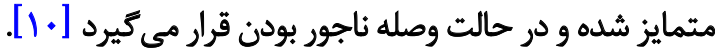

انكَ داراي ريشه يونانى است. در آن زمان جهت ممانعت از فرار برار

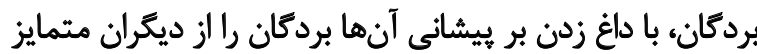

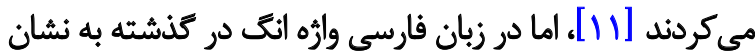

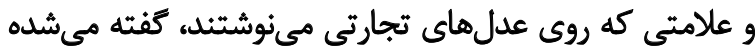

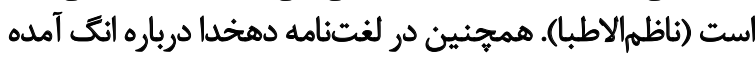

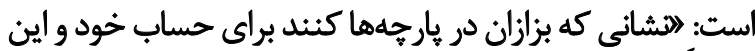

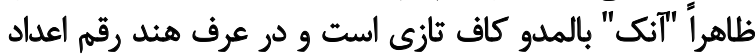

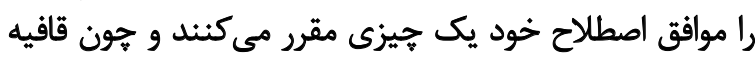

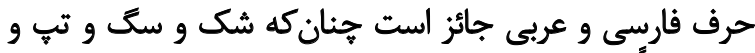

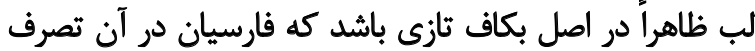

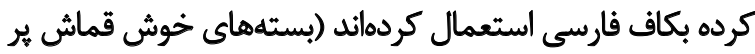

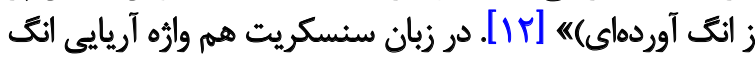

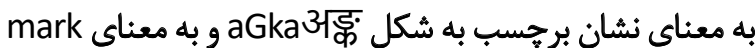

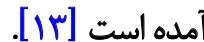

در طول ثاريخ، بيمارىهاى روانيزشكى به طور مداوم با

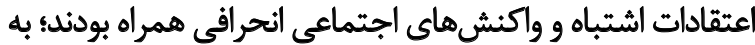

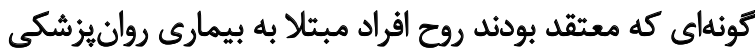

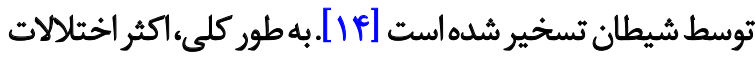

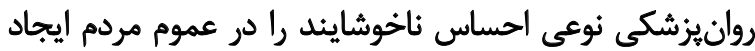

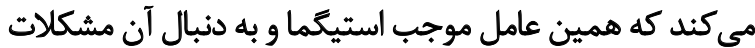

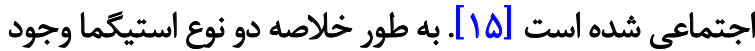

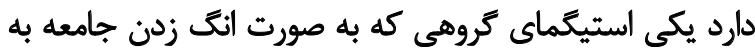

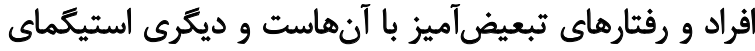

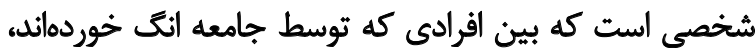

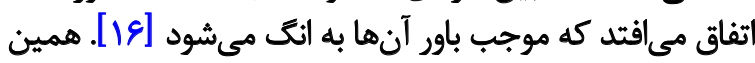

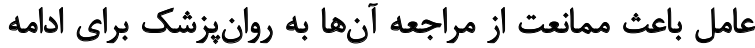

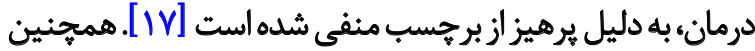

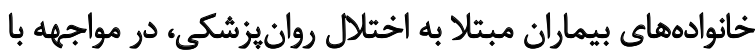

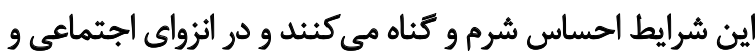

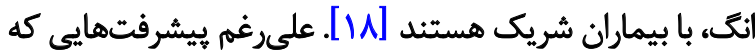

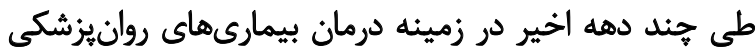

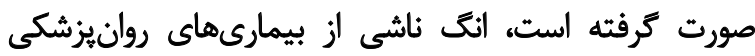

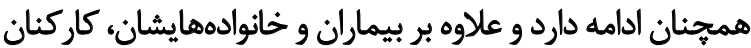

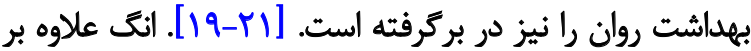




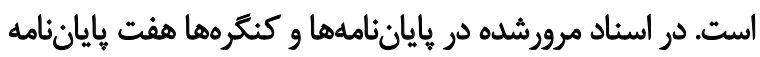

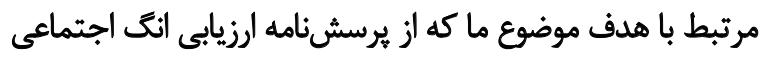

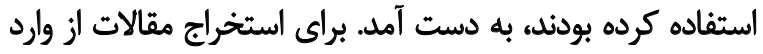

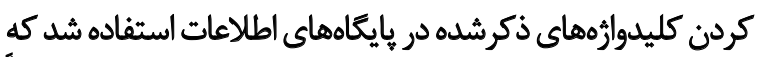

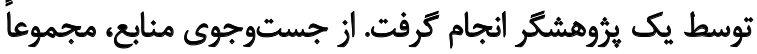

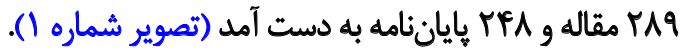

دو نفر از بروهشگران جداكانه مرور هر مقاله و واياياننامههاى

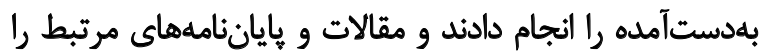

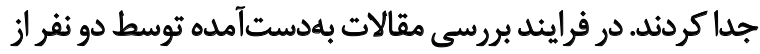

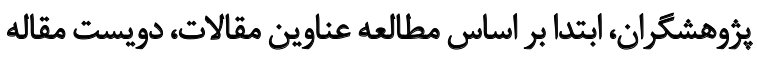

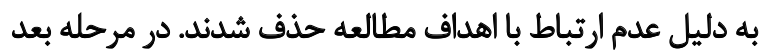

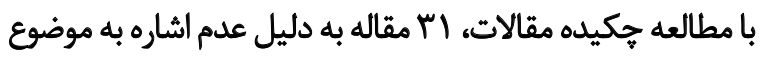

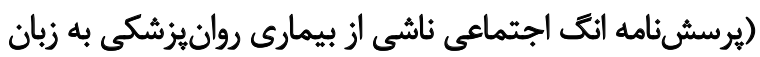

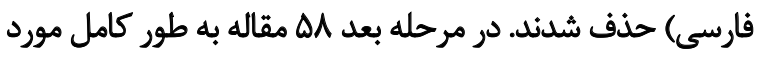

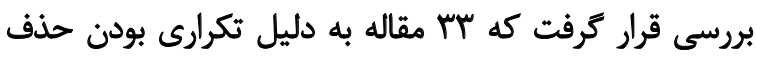

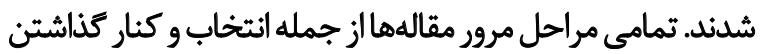

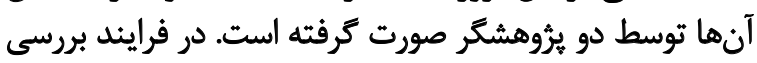

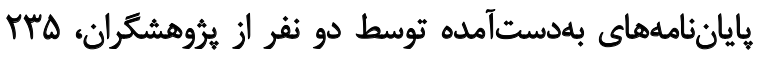

با استفاده از سياهه PRISMA از خايكاههاي اطلاعاتى موجود در

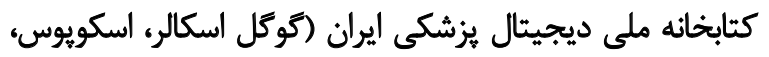

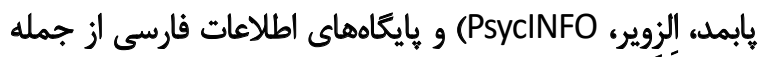

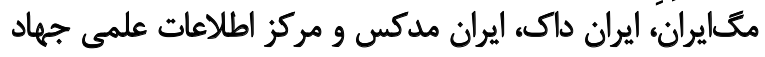

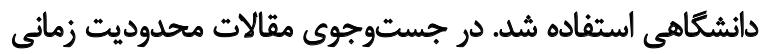

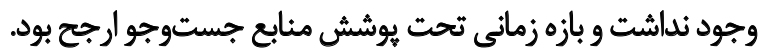

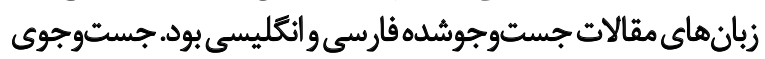

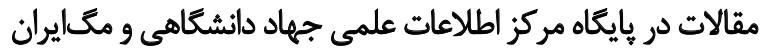

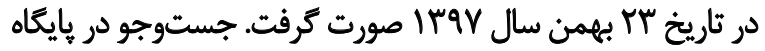

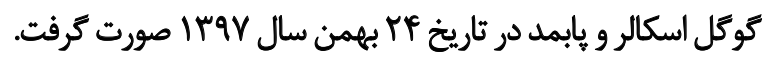

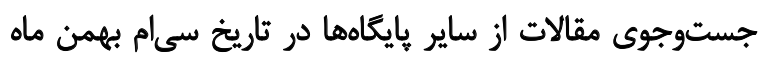

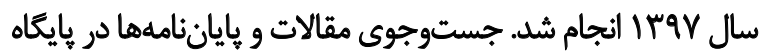

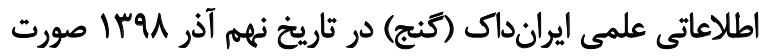

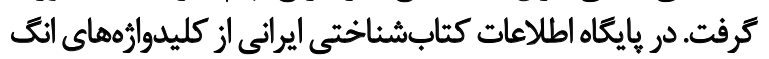

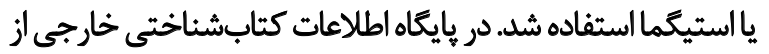

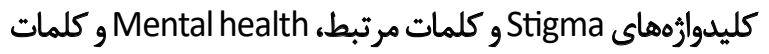

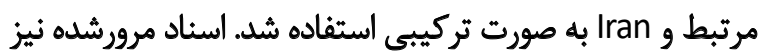
شامل مقالات منتشرشده در مجلات و ارائهشده در كنكرهها بودئه

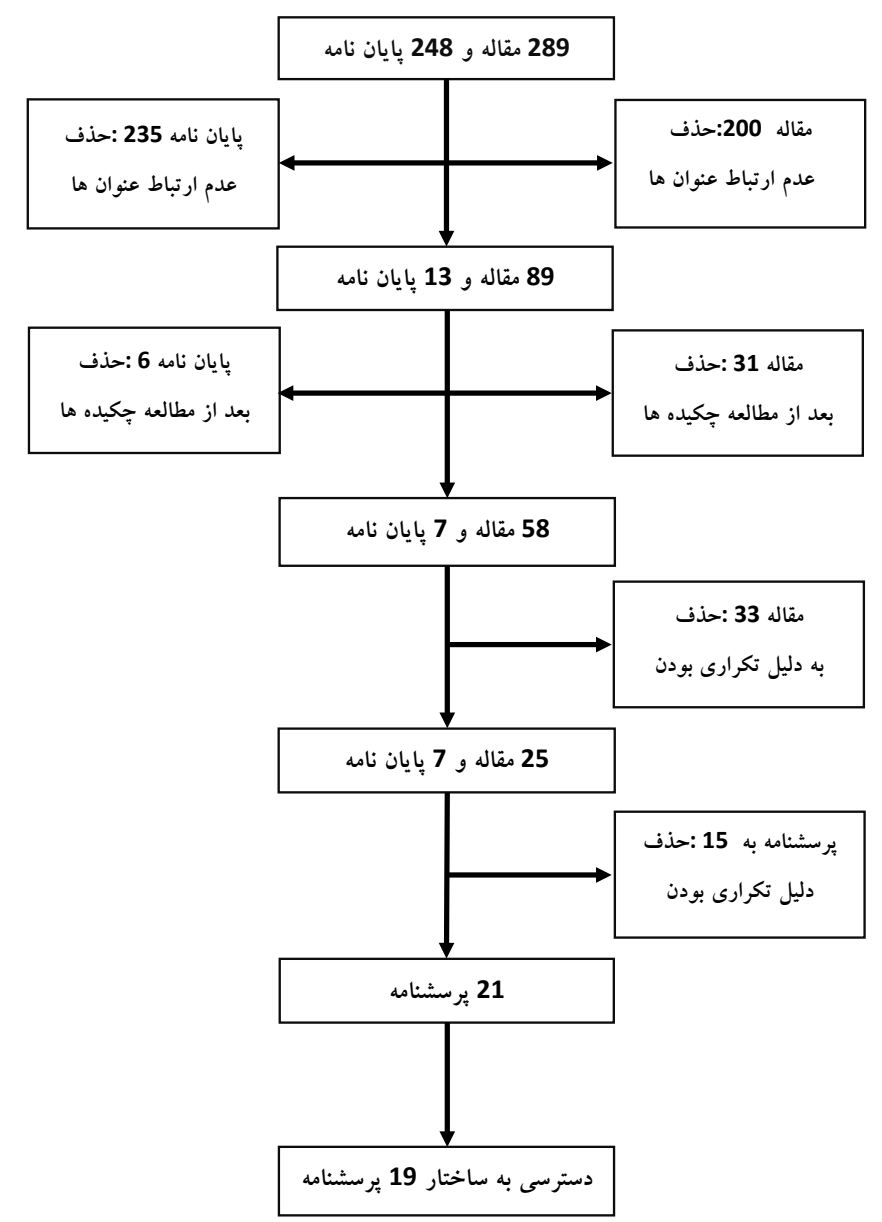




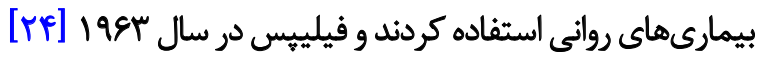

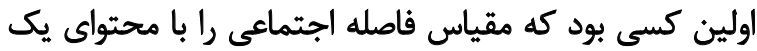

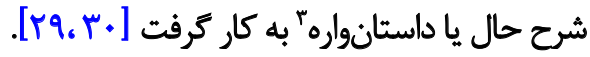

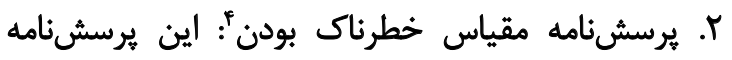

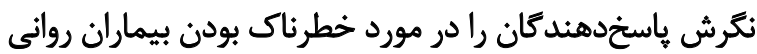

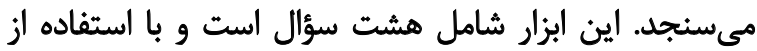

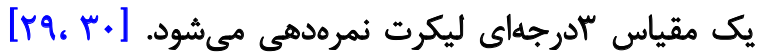

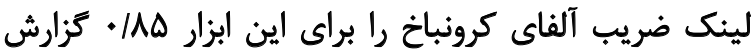
داد [باد

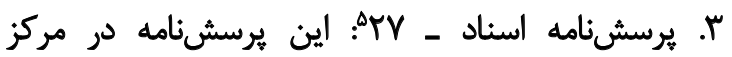

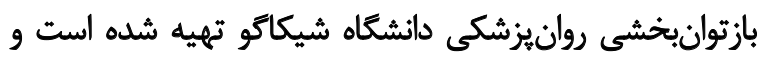

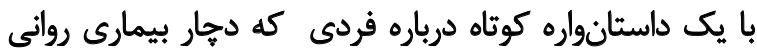

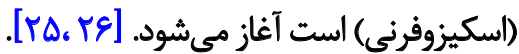

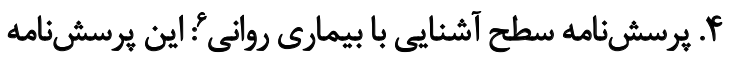

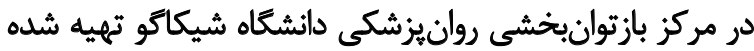

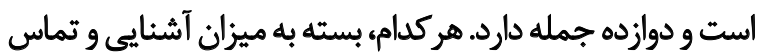

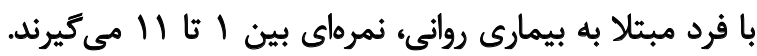

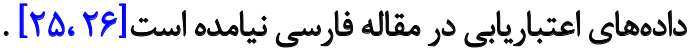
ه. يرسشنامه سنجش نكرش به درمان جويى از متخصصان

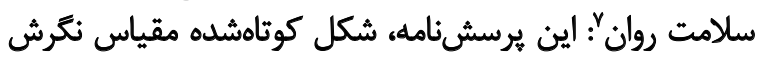

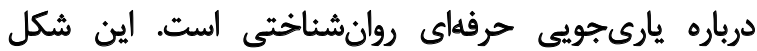

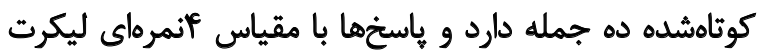

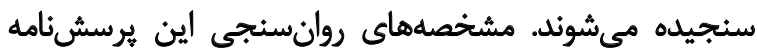

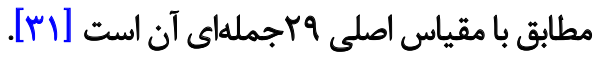
9. يرسشنامه طرح تحقيقاتى بررسى آتاهى و نكرش در مورد

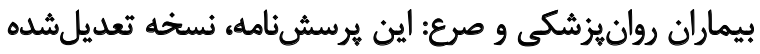

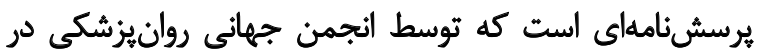

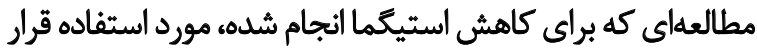

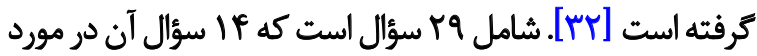

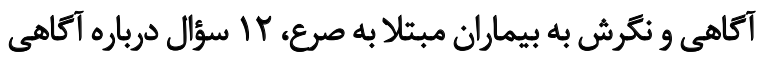

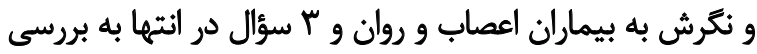

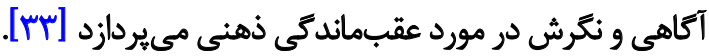

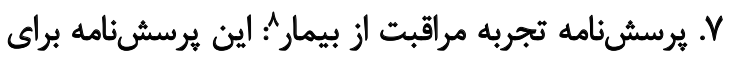

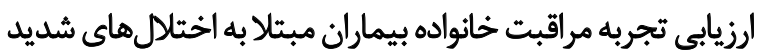
روانيزشكى، همجنين براى ارزيابى ميزان اثربخشى آموزش

\section{Vignette}

4. Perceived Dangerousness of Mental Patients Item (PDMPI)

5. The Attribution Questionnaire (AQ)

6. The Level of Familiarity Scale (LFS)

7. Attitudes toward Seeking Professional Psychological Help (ASPPH)

8. Experience of Caregiving Inventory

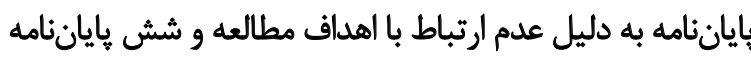

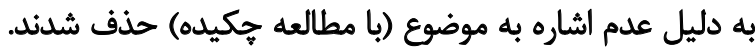

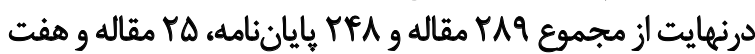

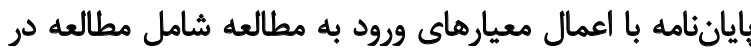

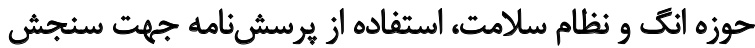

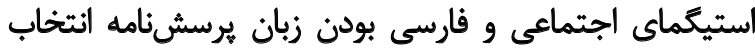

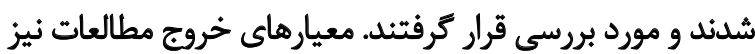

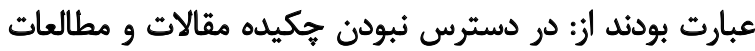

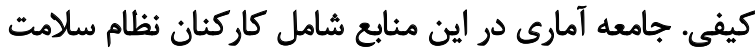

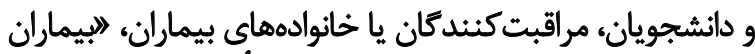

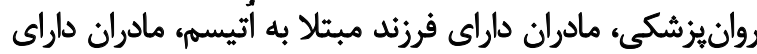

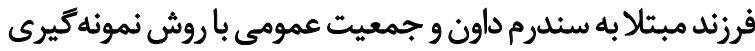
تصادفى يا نمونه كيرى در دسترس بود جمعيد

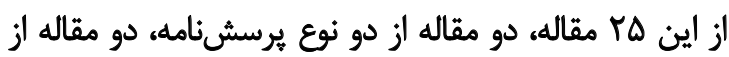

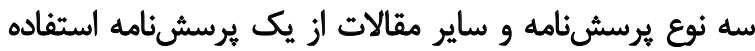

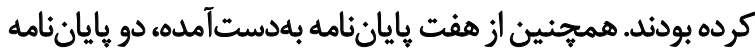

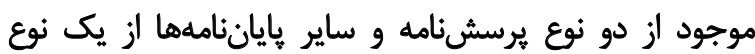

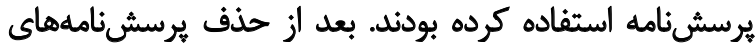

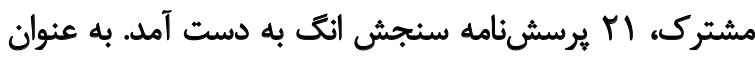

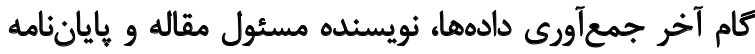

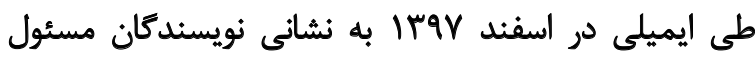

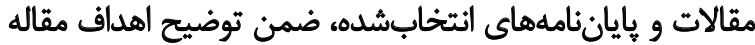

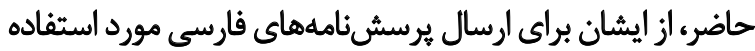

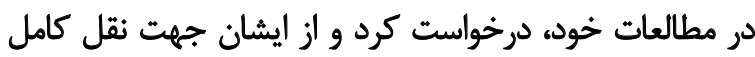

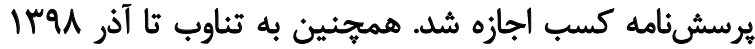

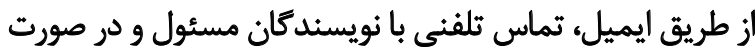

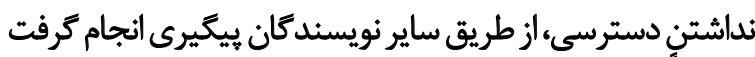

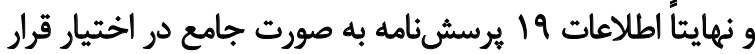

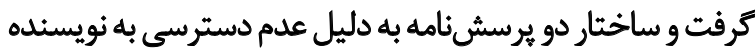

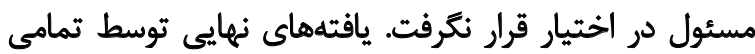

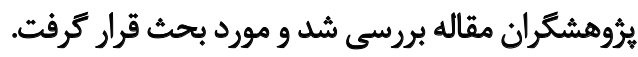

ياقتهها

آب برسشنامه بهكار فتته در مقالات بهدستآمده در ايران، در

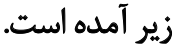

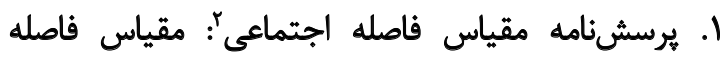

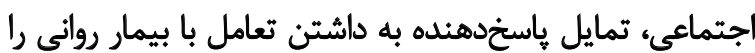

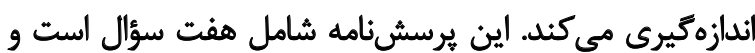

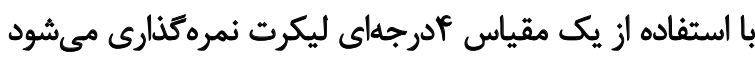

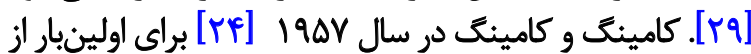

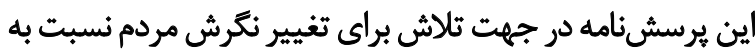




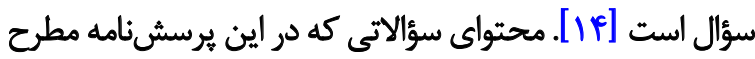

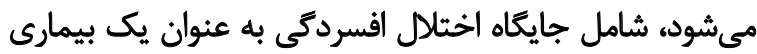

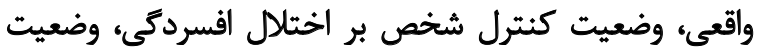

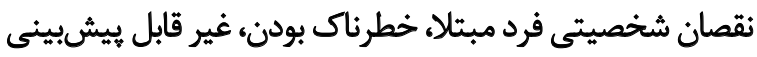

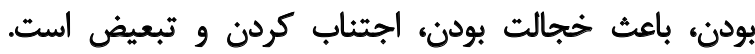

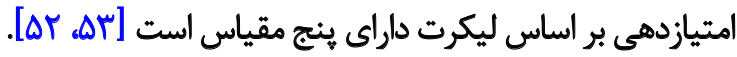

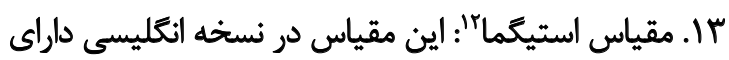

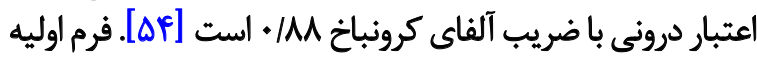

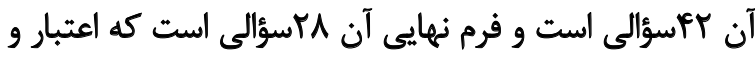

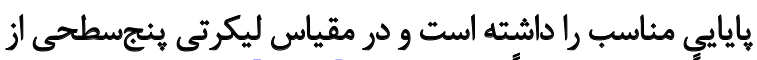

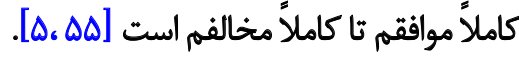

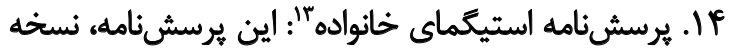

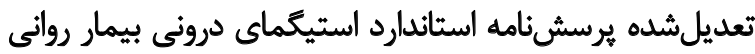

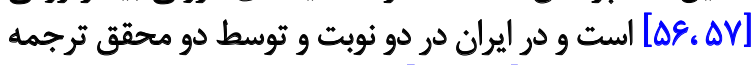

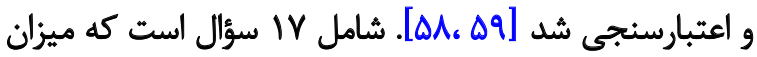

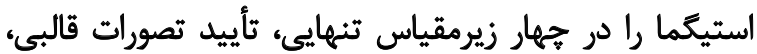

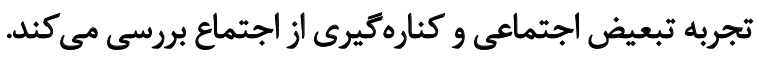

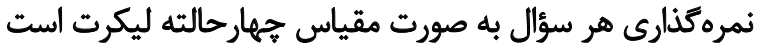

[०१]

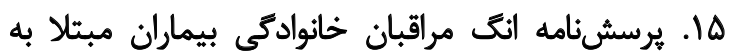

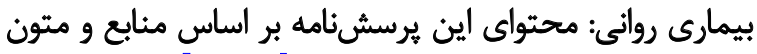

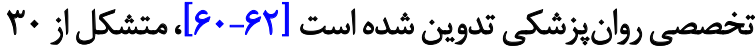

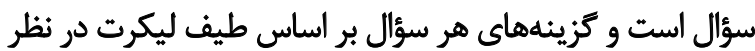
كرفته شده است است كزينهائ.

18. يرسش نامه استيكماى فراهم كنيدكان مراقبت بهداشتى "1 اين يرسش لينامه شامل بيست جمله بوده كه بامقياس ينجحالته

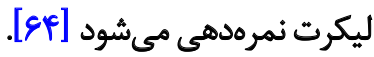

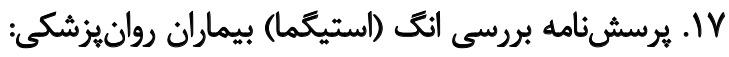

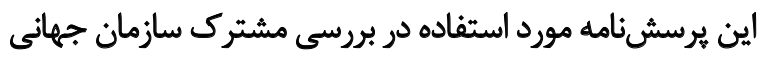

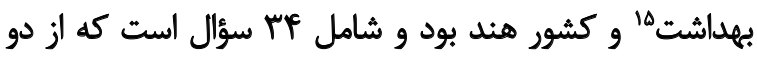

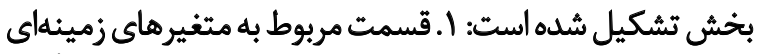

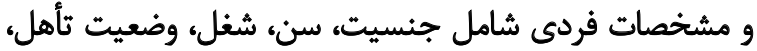

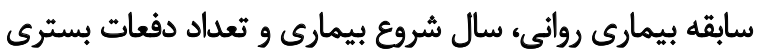

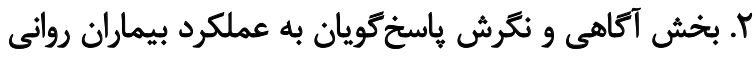

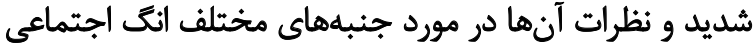
بيمارى [ـهابد.

11. يرسش نامه انك دريافتشده: اين يرسش نامه توسط محقق

12. Stigma Scale (SS)

13. The Parents Internalized Stigma of Mental Illness Scale (PISMI) 14. The Opening Minds Scale for Health Care (OMS-HC) Providers 15. World Health Organization (WHO)

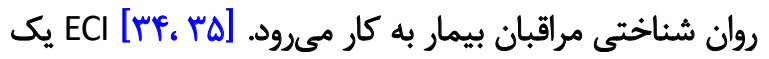

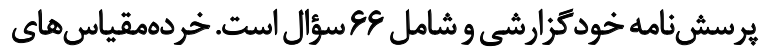

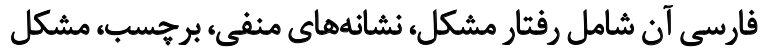

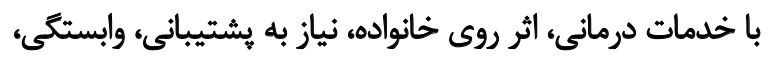

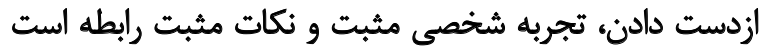

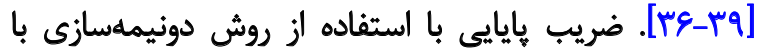

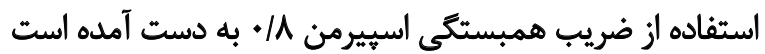

[. [F.]

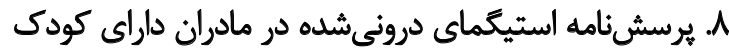

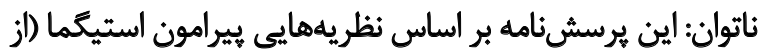

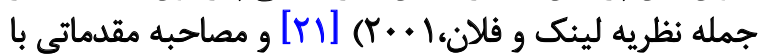

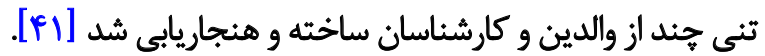

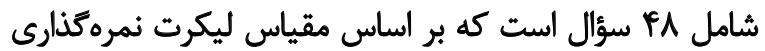

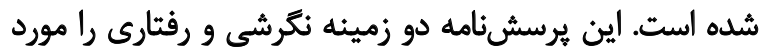

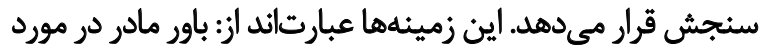

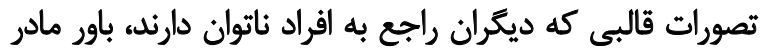

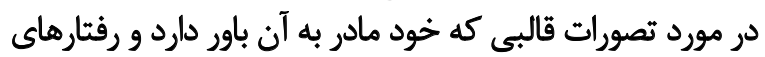

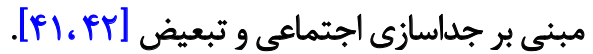

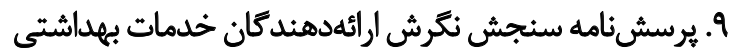

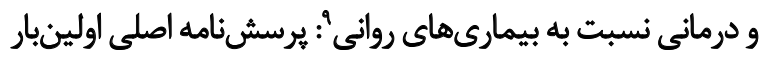

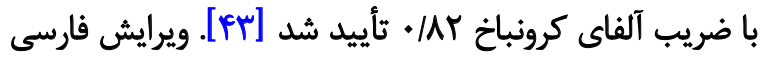

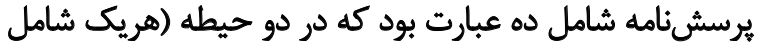

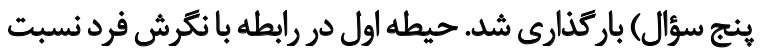

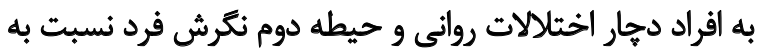

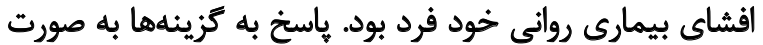

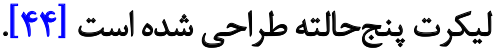

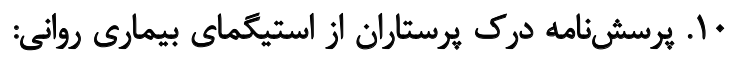

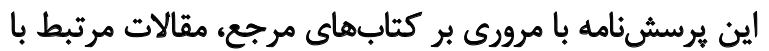

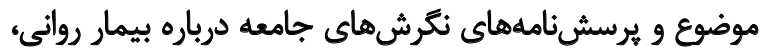

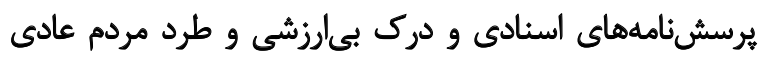

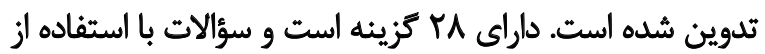

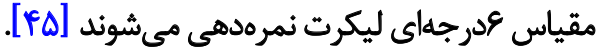

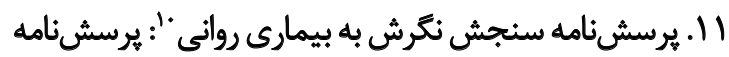

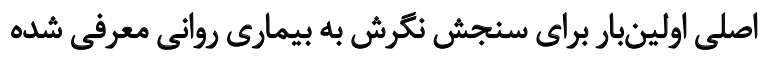

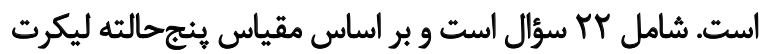

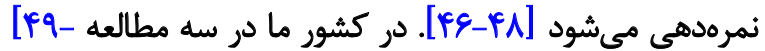

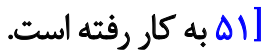

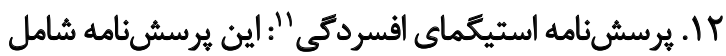

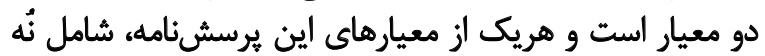

9. Opening Minds Scale for Health Care Providers (OMS-HC) 10. Opinion about Mental Illness (OMI)

11. Depression Stigma Scale (DSS) 


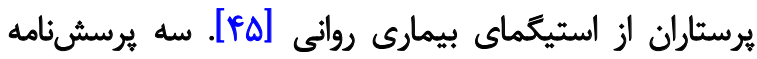

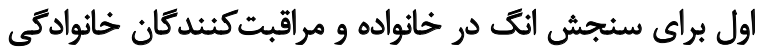
بيماران طراحى شدهاند.

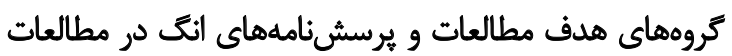

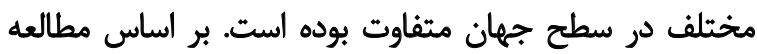

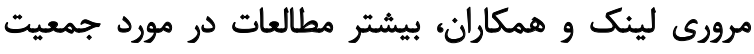

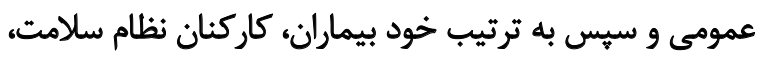

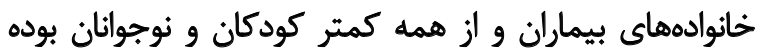

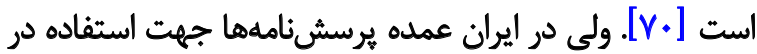

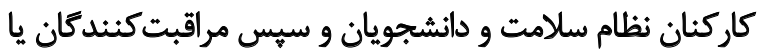

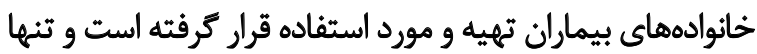

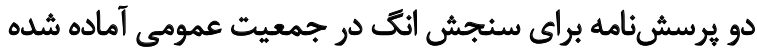

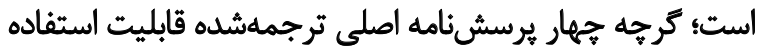

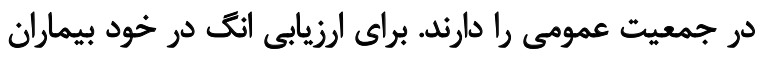

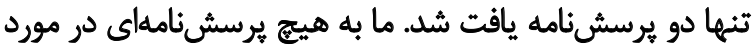

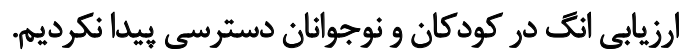

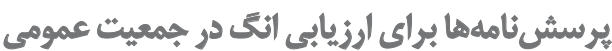

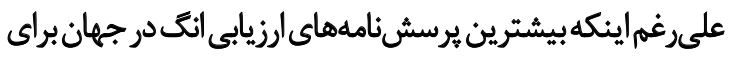

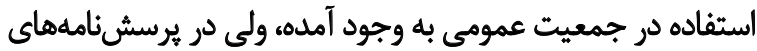

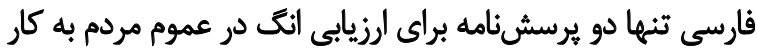

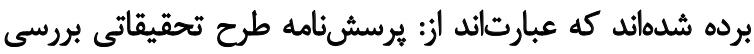

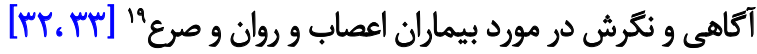

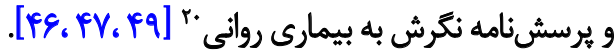

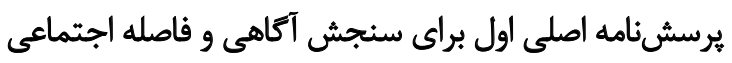

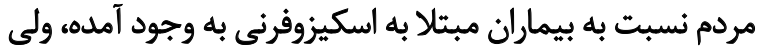

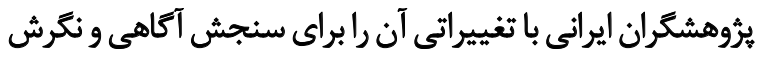

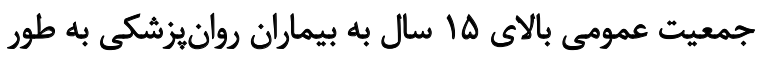

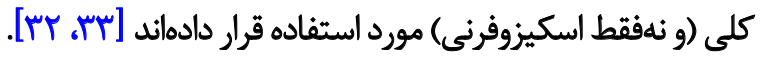

برسشنامه اصلى دوم بالعكس براى سنجش نترش

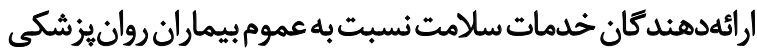

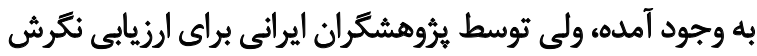

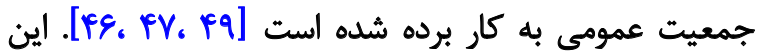

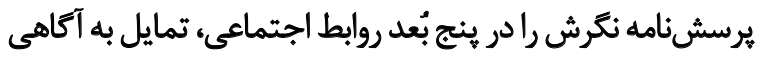

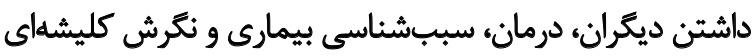

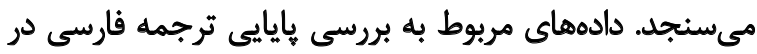

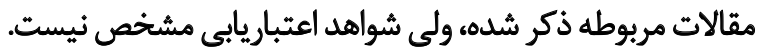
يرسشنامهاى اصلى مقياس فاصله اجتماعى" و مقياس

19. Telephone Interviewers followed a Computer-generated, Structured Questionnaire (TI-CSQ)

20. Opinion about Mental IIIness (OMI)

21. Social Distance Item (SDI)

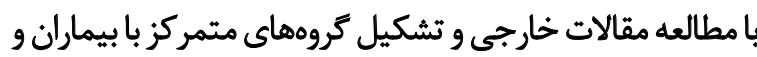

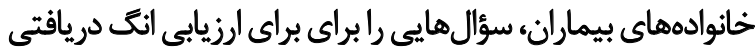

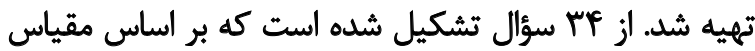

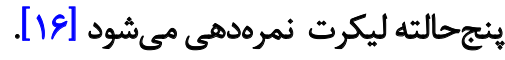

19. يرسشنامه ارزيابى شناختى استرس مرتبط با انكَ"ا.

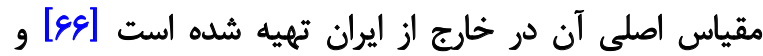

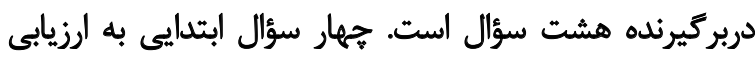

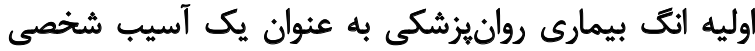

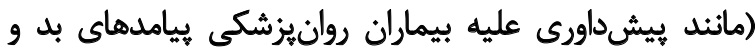

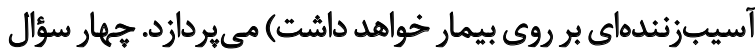

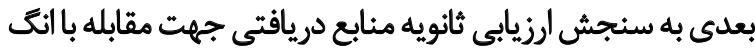

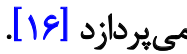

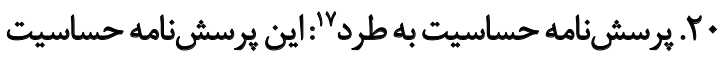

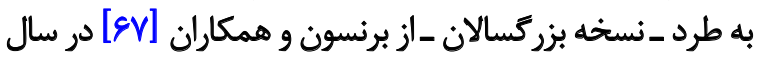

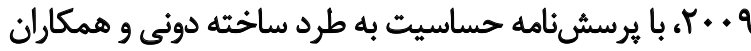

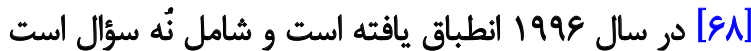

ا r. يرسش نامه انك ادراكشده والدينى": مقياس اصلى اين

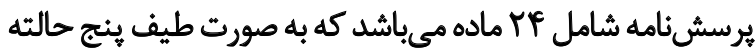

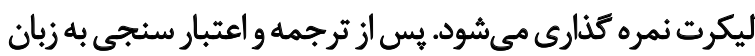

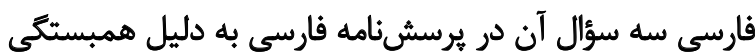

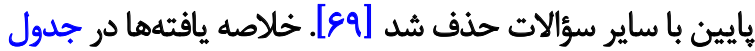
شماره آمده است.

بحث

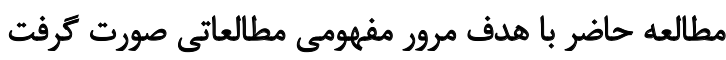

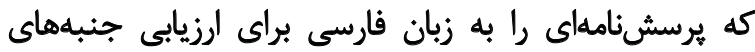

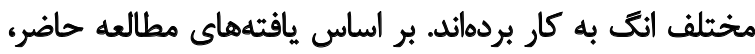

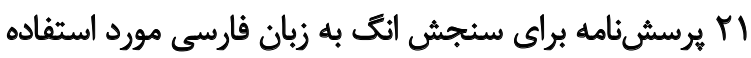

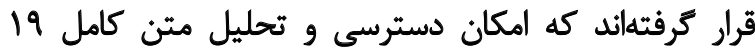

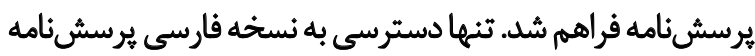

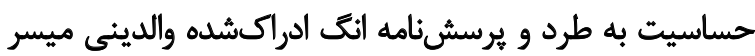

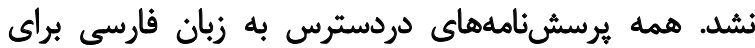

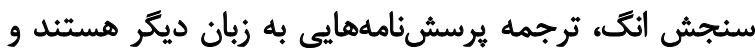

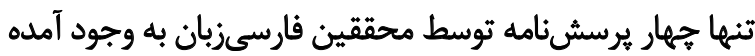

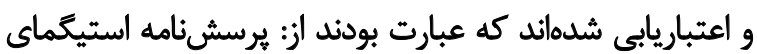

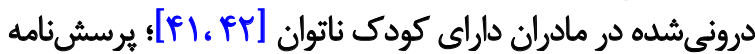

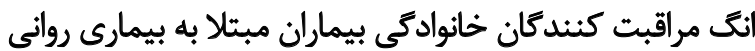

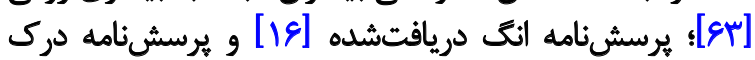

16. Cognitive Appraisal of Stigma-related Stress (CASS)

17. Rejection Sensitivity Questionnaire (RSQ)

18. Perceived Stigma Scale - Revised 


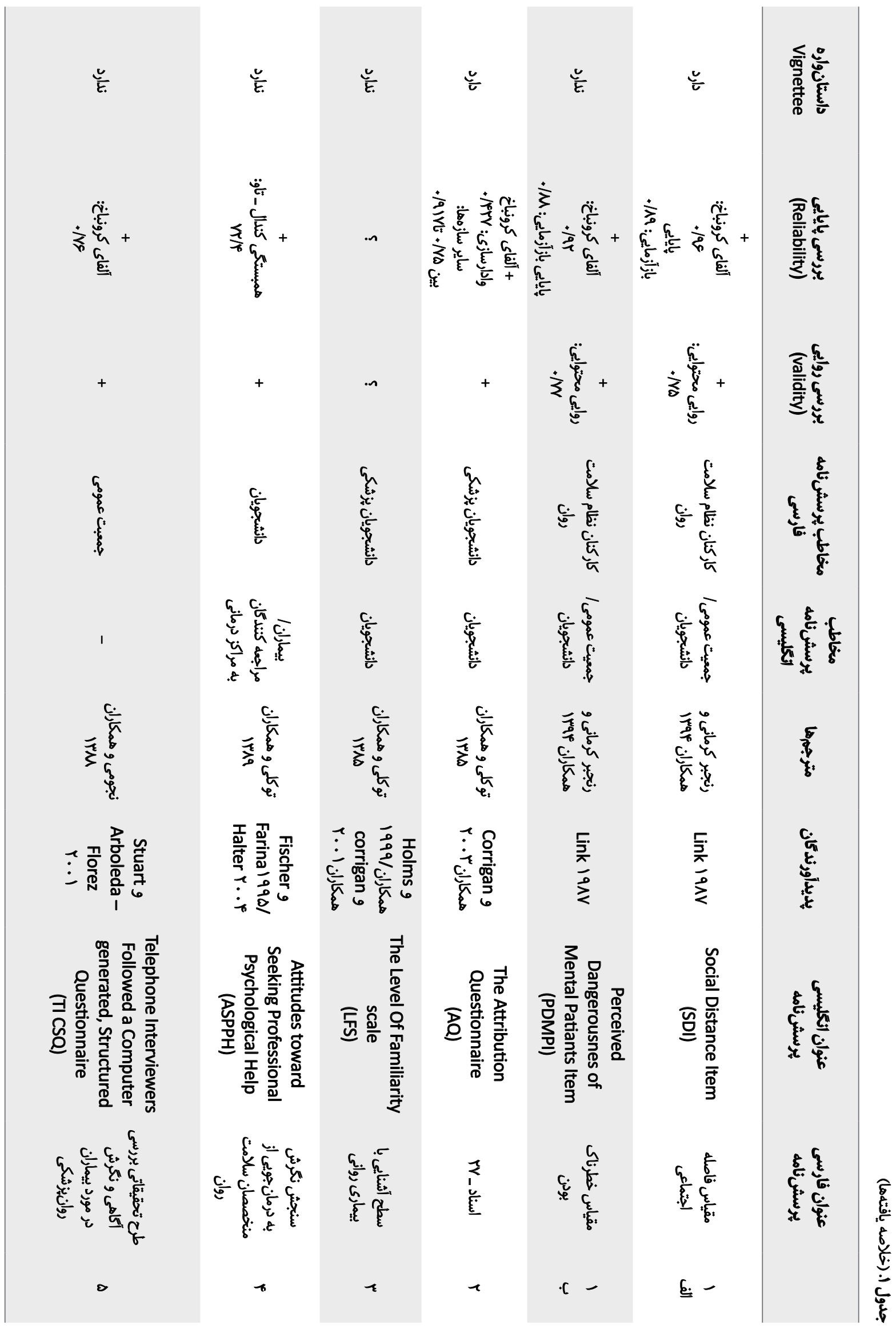




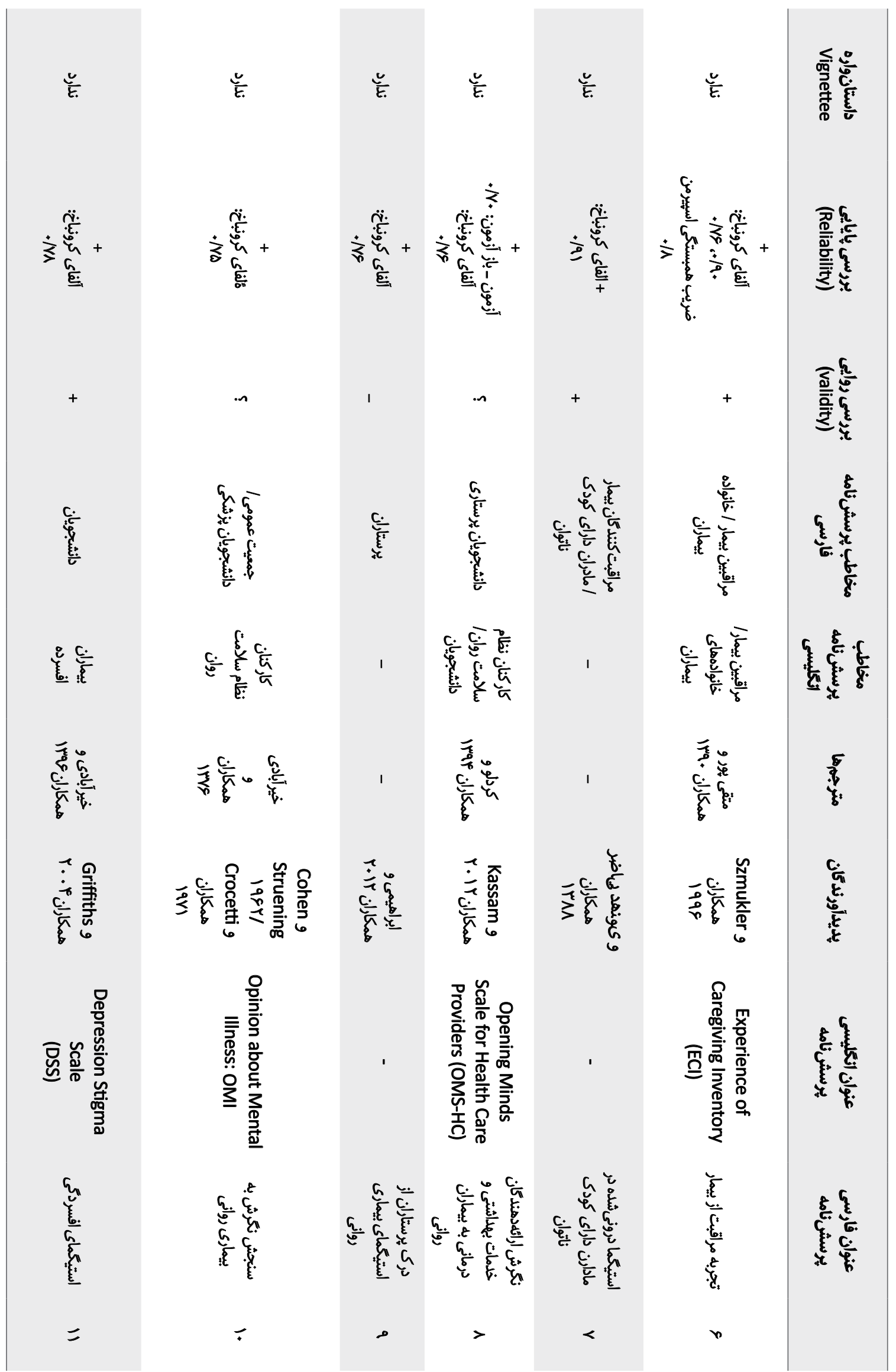




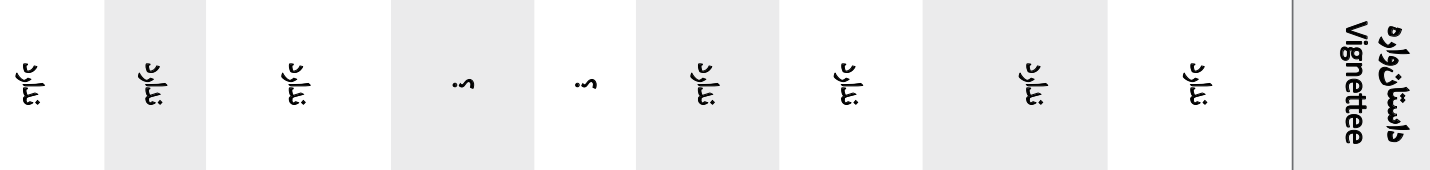

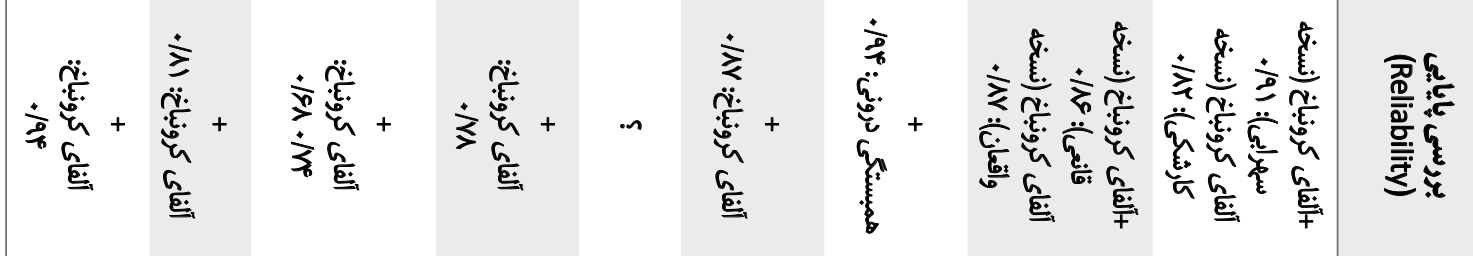

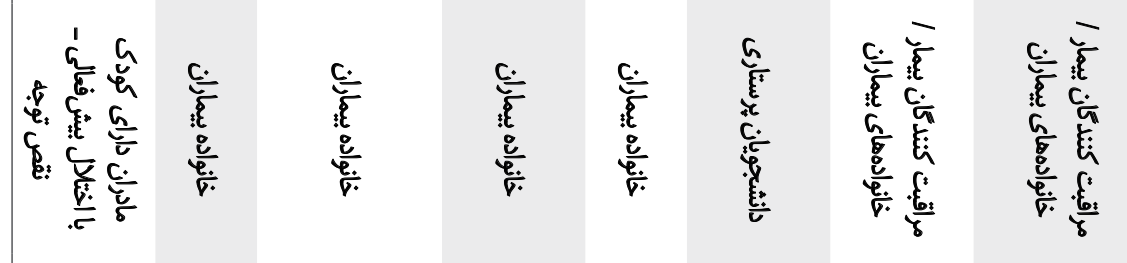

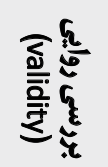

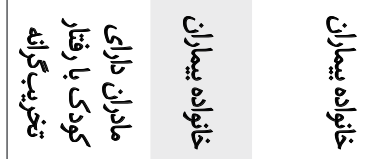

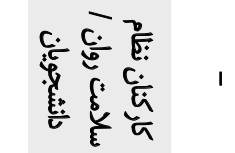

है

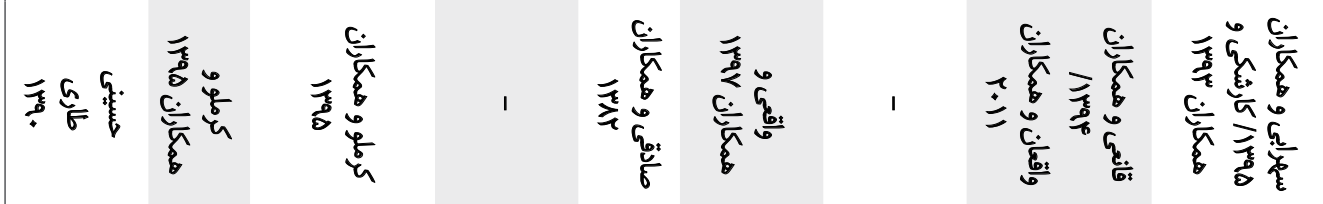

$\frac{5}{5}$

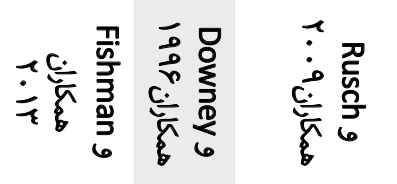

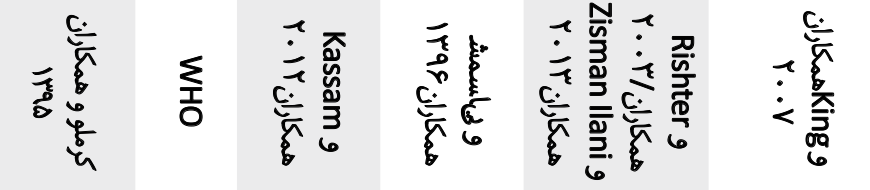

$\frac{c}{c}$

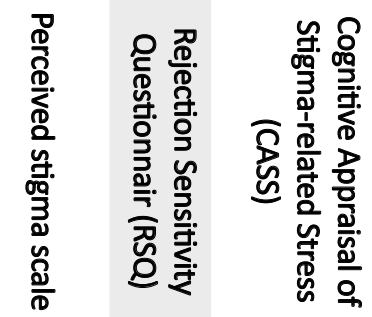

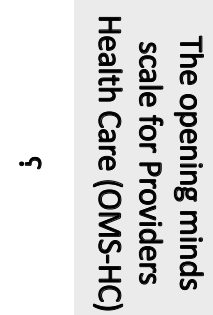

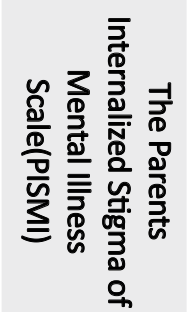

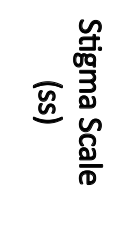

$\frac{\xi}{\frac{c}{6}}$

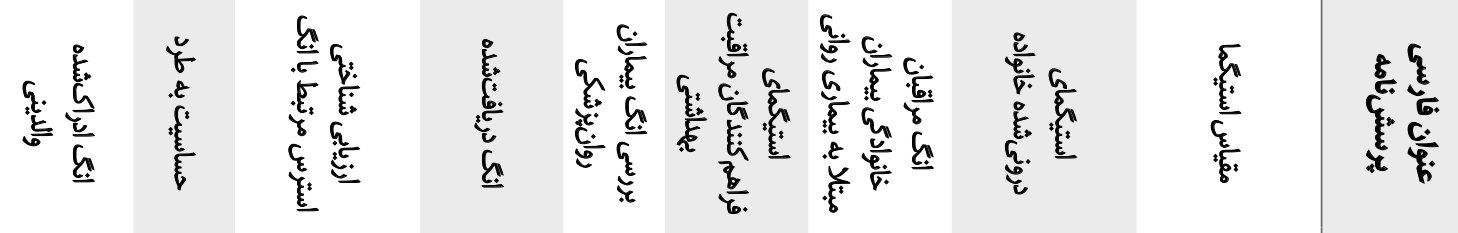




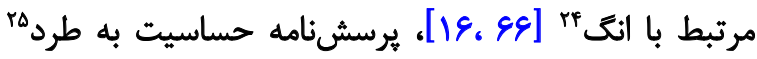
روان [19، 9V، 9N]

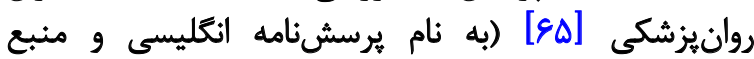

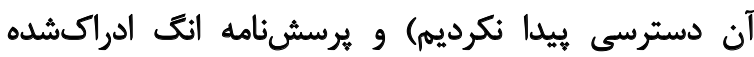

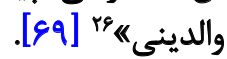

يرسشنامه تجربه مراقبت از بيمار براى استفاده در

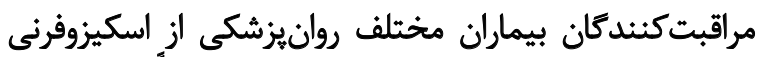

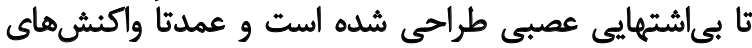

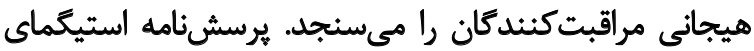

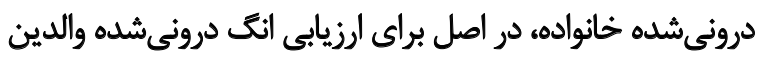

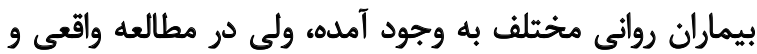

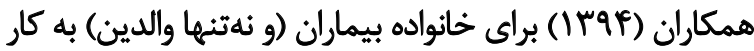

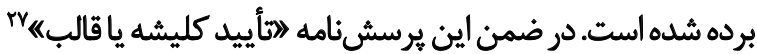

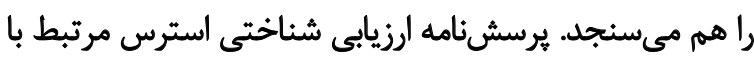

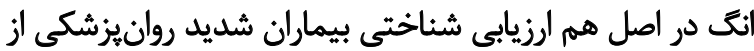

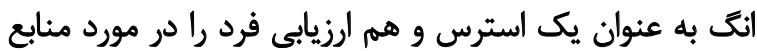

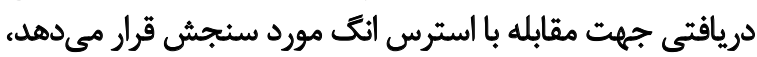

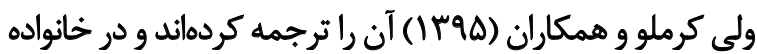
بيماران شديد روانيزشكى به كار بردهاند.

با توجه به اينكه يرسش نامه اصلى براى بيماران طراحي شدائه

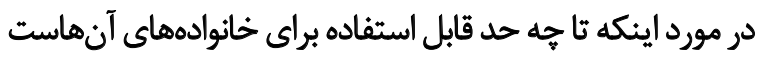

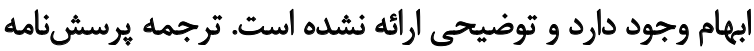

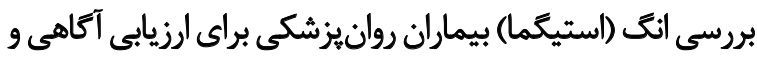

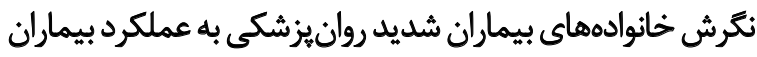

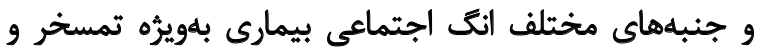

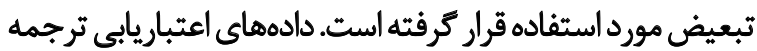

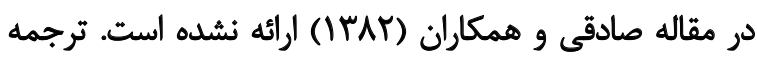

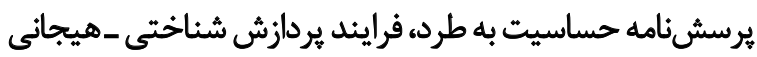

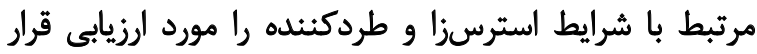

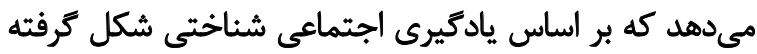

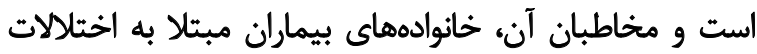

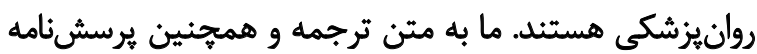

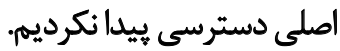

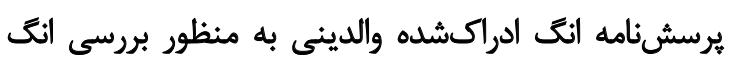

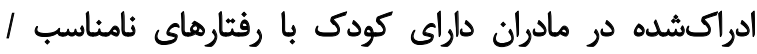

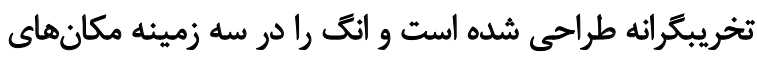

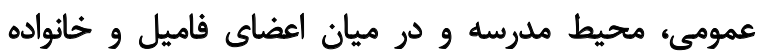

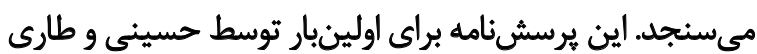

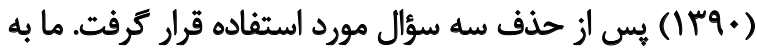

24. Cognitive Appraisal of Stigma-related Stress (CASS)

25. Rejection Sensitivity Questionnaire (RSQ)

26. Perceived Stigma Scale - Revised

27. Stereotype endorsement
خطرناك بودن بار براى ارزيابى در جمعيث عمومى به وجود آماده

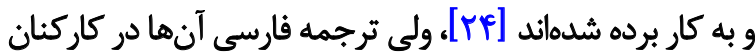

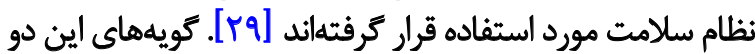

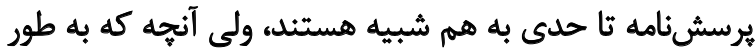

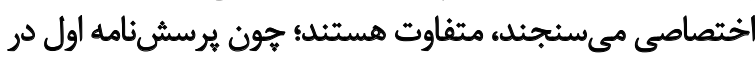

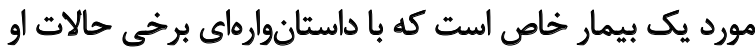

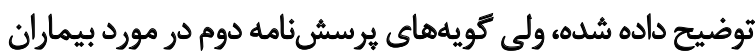

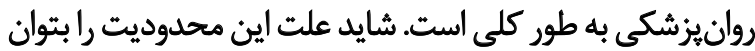

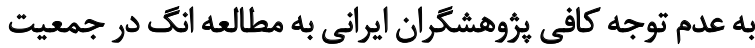

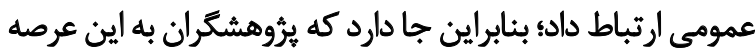

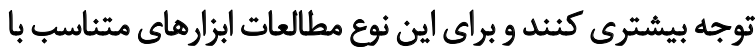

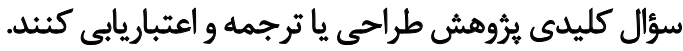

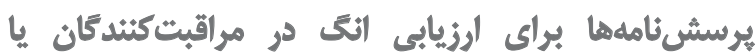 خانوادههاي بيمار ان روان بئشيكي}

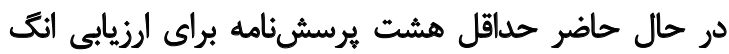

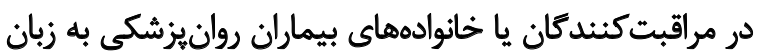

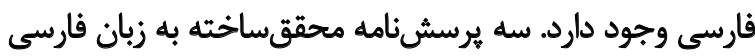

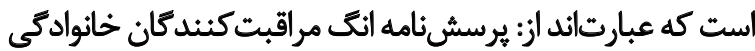

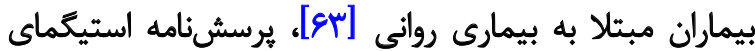

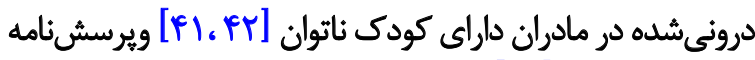

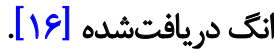

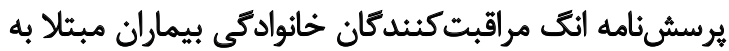

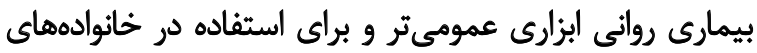

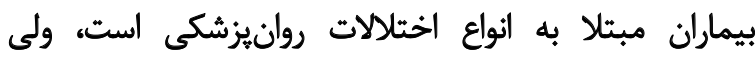

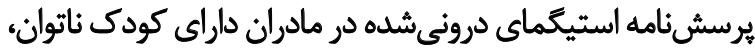

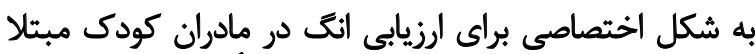

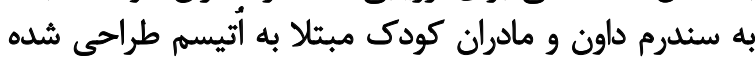

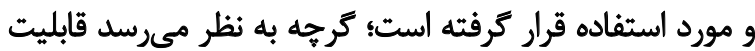

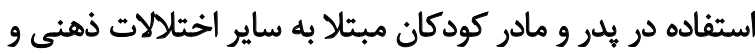

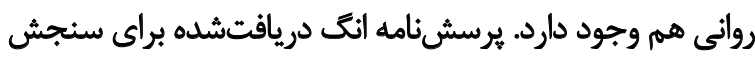

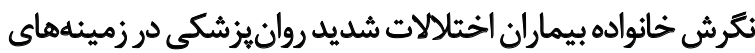

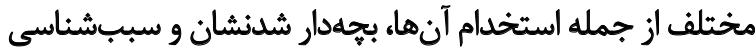
رُنتيكى بيمارى آنها طراحى شده است.

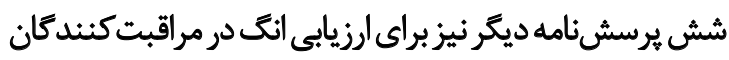

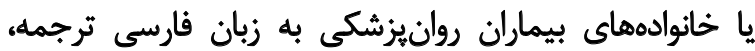

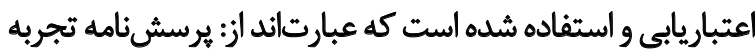

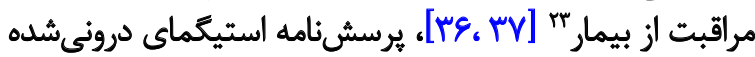

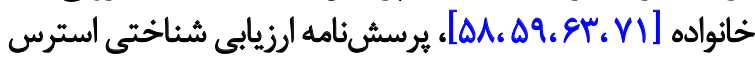

22. Peceived Dangerousness Of Mental Patiants Item (PDMPI) 23. Experience of Caregiving Inventory (ECI) 


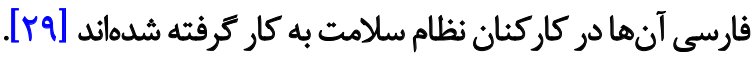

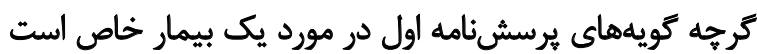

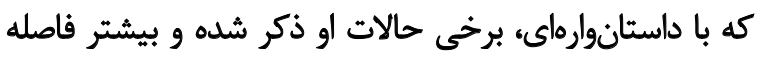

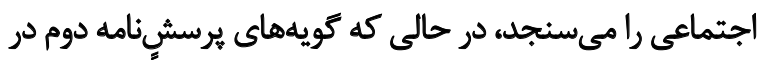

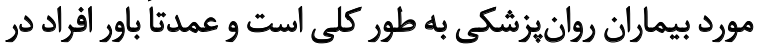
مورد خطرناك بودن اين بيماران را الرزيابى مى كند.

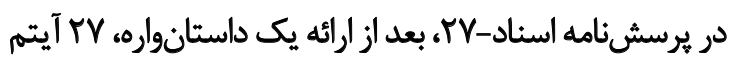

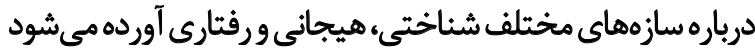

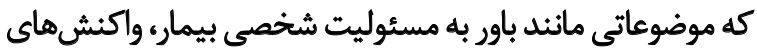

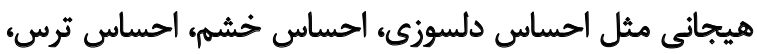

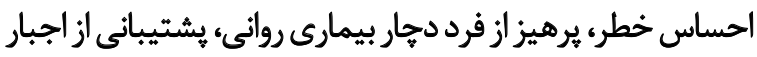

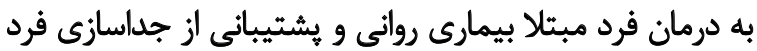

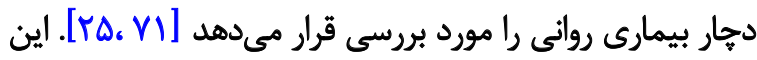

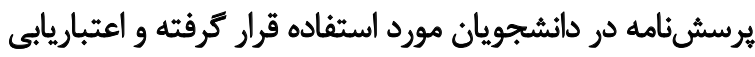
شده است.

و يرسش تامه سطح آشنايى با بيمارى روانى، براى ارزيابى آكاهى باهي

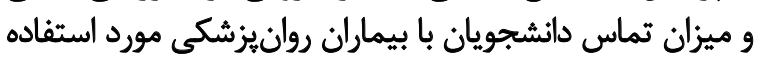

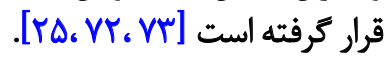

يرسشنامه سنجش نكرش به درمان جويى از متخصصان

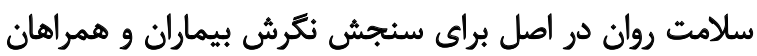

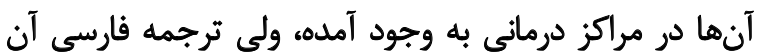

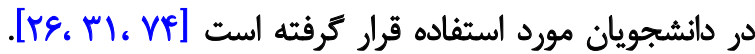

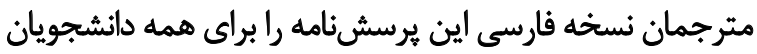

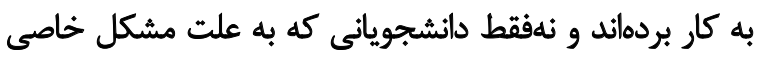
متقاضى كمك حرفهاى بودهاند.

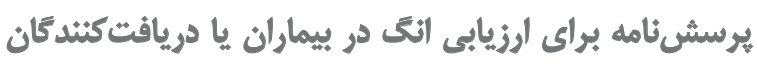
colli

ما تنها به دو يرسشنامه جهت سنجش انق در خود بيماران

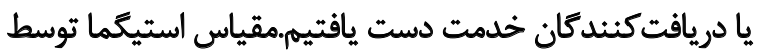

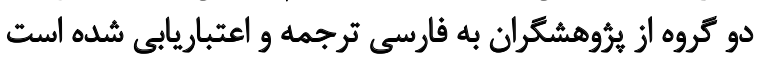

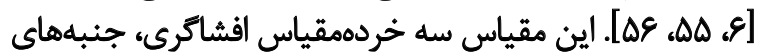

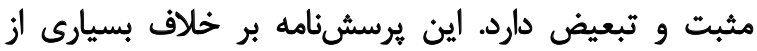

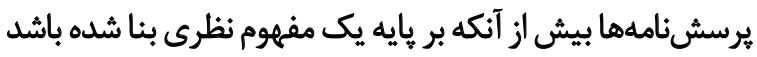

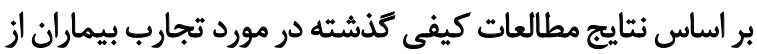

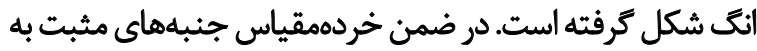

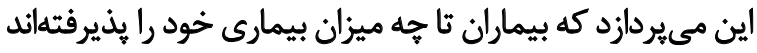

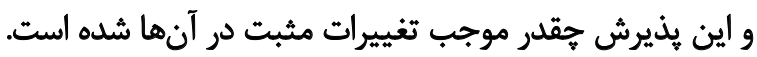

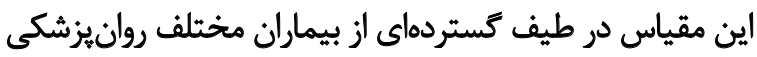

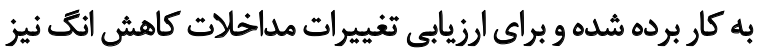
مى توان از آن استفاده كرد.

يرسشنامه استيكماى افسردكى براى سنجش انك در بيماران

\author{
متن يرسشنامه فارسى دسترسى ييدا نكرديم.

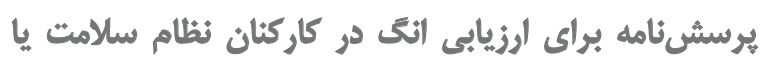

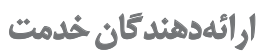

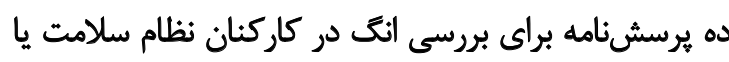

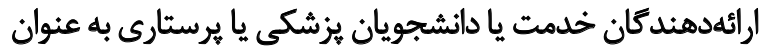

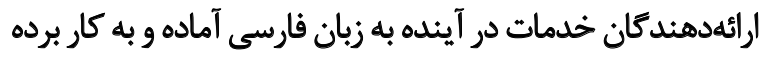

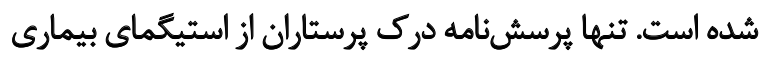

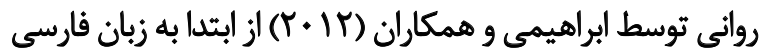

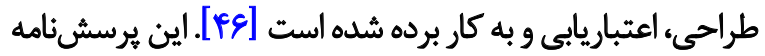

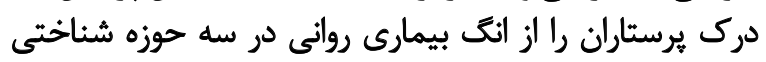

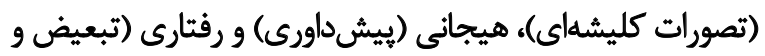

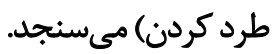

Opening Minds Scale for Health Care ترسشنامنه (Providers (OMS-HC

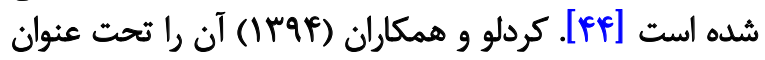

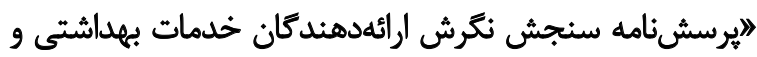

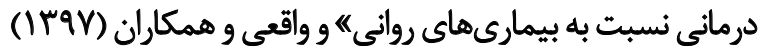

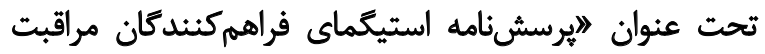

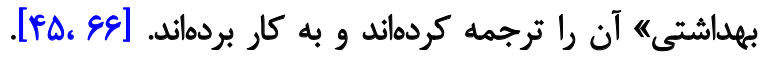

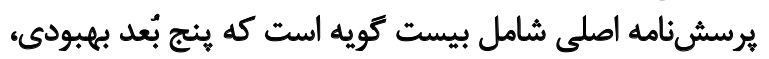

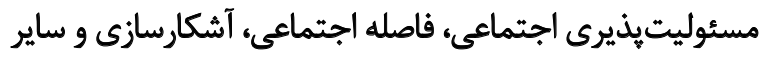

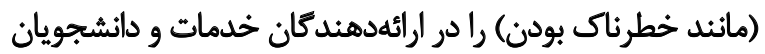

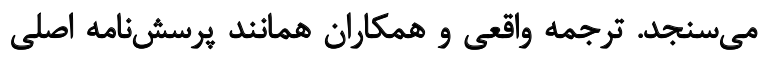

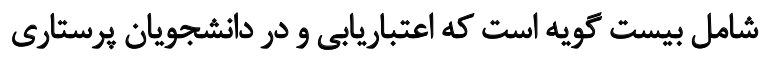

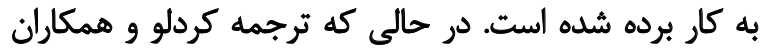
تن (I Tqf)

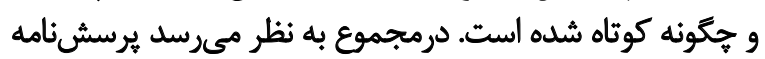

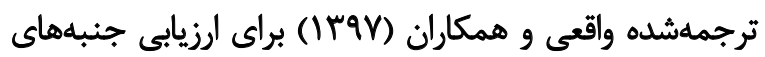

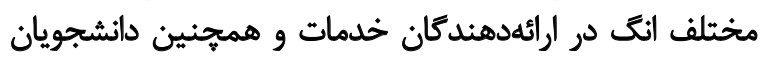

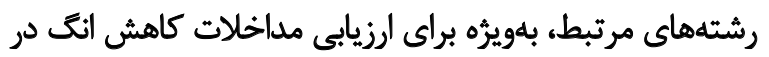
آن ها بسيار مناسب باشد.

يرسش ائامه سنجش نكُش به بيمارى روانى نيز براي ارزيابي

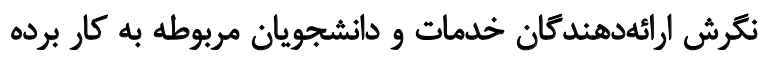

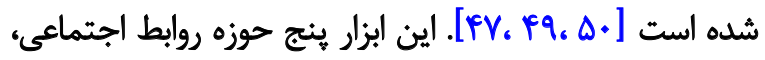

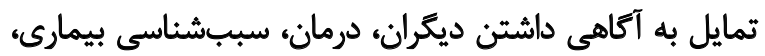

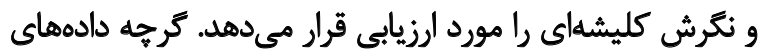

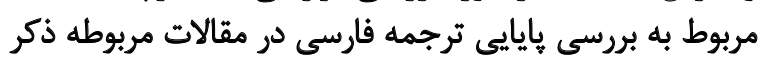

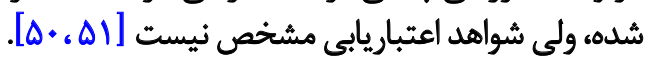

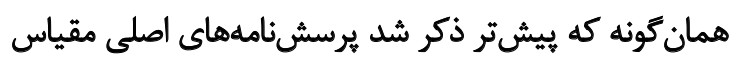

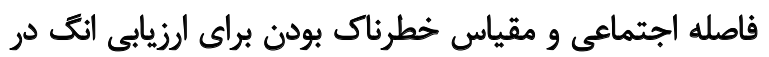

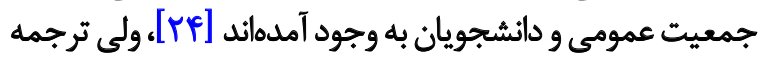




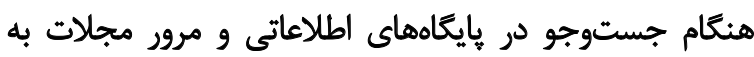

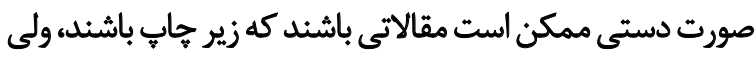

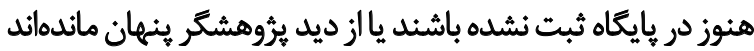

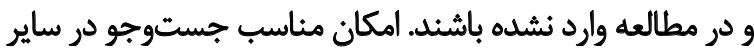

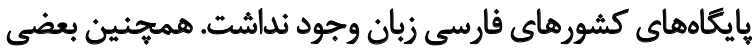

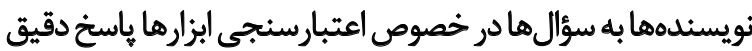

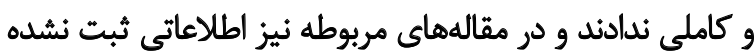

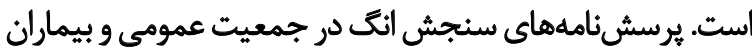

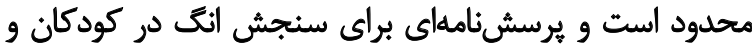
نوجوانان به زبان فارسى يافت نشد.

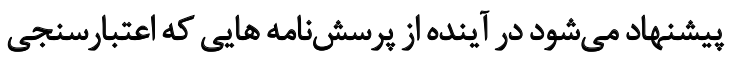

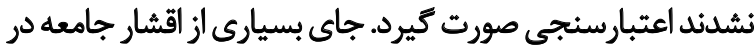

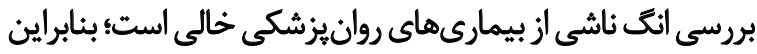

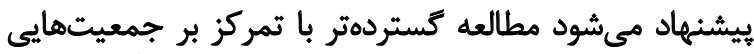

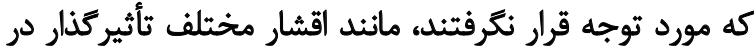

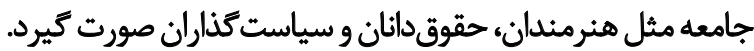

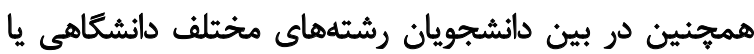

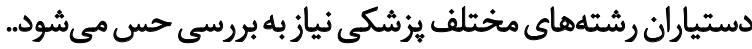

\section{تثيجليَّيرى}

برخلاف آنجه در جهان اتفاق افتاده (بيشتر برسشنامههاى

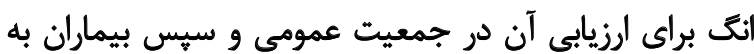

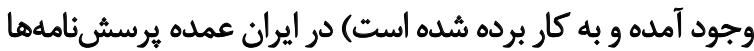

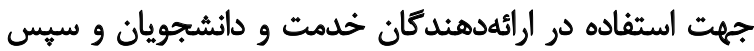

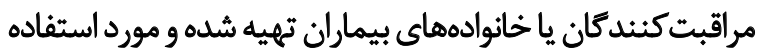

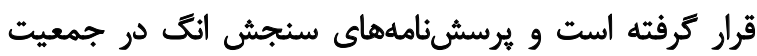

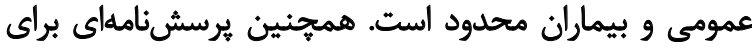

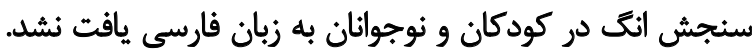

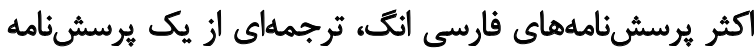

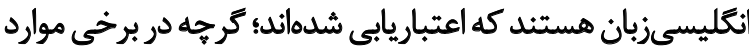

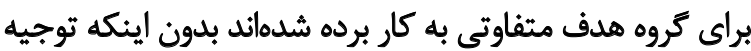
آن بلهخوبى تبيين شده باشد.

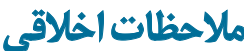

\section{ييروى أز الصول اخلاق بثؤهش}

بابت استفاده از برسشنامهها از تمامى نويسندكان از طريق بازيق

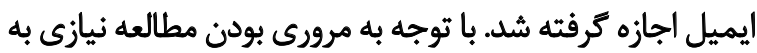

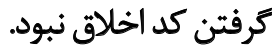

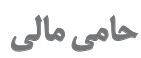

اين تحقيق هيج كونه كمك مالى از سازمانهاي تأمين مالى در بخشهاى عمومى ، تجارى يا غير ائتفاعى دريافت نكردي
افسرده به وجود آمده است و دو عامل استيكماي شخصى، يعنى

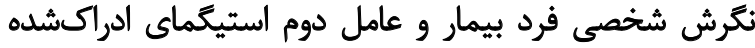

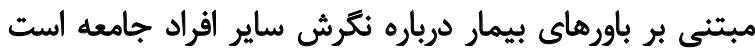

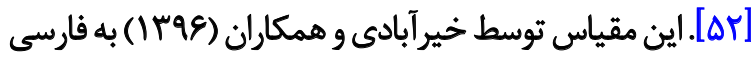

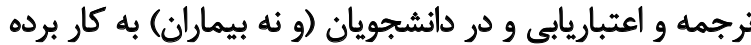

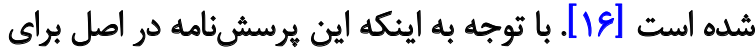

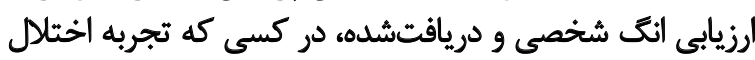

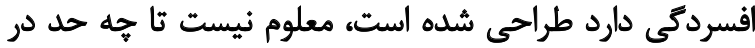

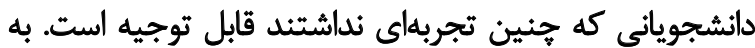

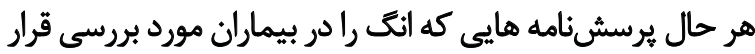
دهند به زبان فارسى بسيار محدودند

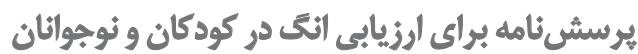

ما به برسشنامهاى كه انك را در كودكان و نوجوانان مورد

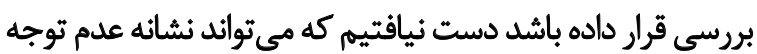
ي بروهشكران به اين حوزه باشد.

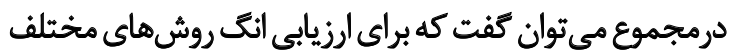

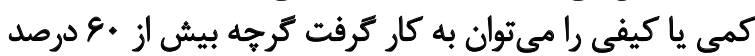

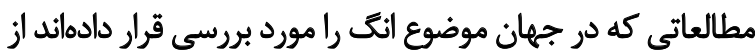

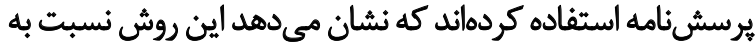

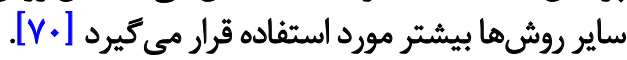
يرسشنامهها معمولاً بر اساس يك ساختار مفهومى شكل

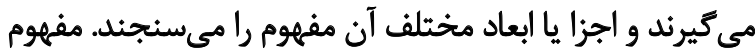

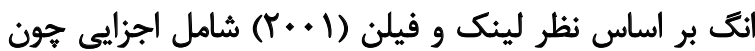

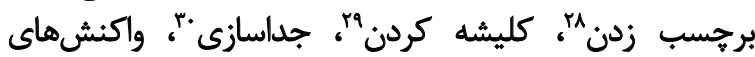

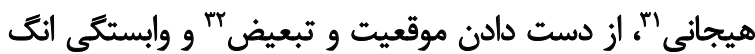

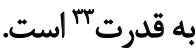

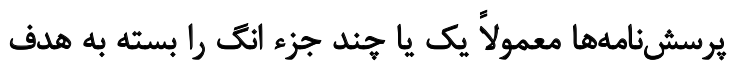

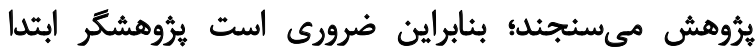

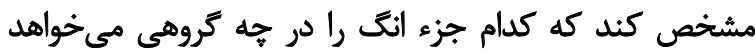

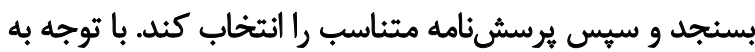

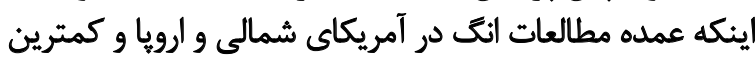

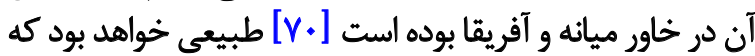

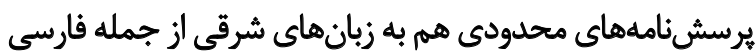

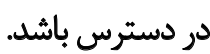

از محدوديتهاى اساسى در اين ثرُوهش اين است كه در

28. Labeling

29. Stereotyping

30. Separating

31. Emotional reactions

32. Status loss and discrimination

33. Dependence of stigma on power 


$$
\text { مشاركت نويسندكّان }
$$

طراحى مطالعه: سيد وحيد شريعت، فرحناز رضوانىفر و فروانيان

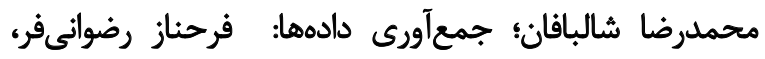

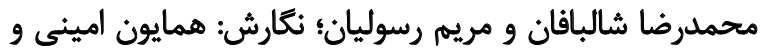
فرحناز رضوانىفرا: ويرايش و بازنويسى مقاله: همه نويسندكان.

$$
\text { تقعار منافع }
$$

بنابر اظهار نويسندكان، اين مقاله تعارض منافع ندارد. 


\section{References}

[1] Rüsch N, Angermeyer MC, Corrigan PW. Mental illness stigma: Concepts, consequences, and initiatives to reduce stigma. European Psychiatry. 2005; 20(8):529-39. [DOI:10.1016/j.eurpsy.2005.04.004] [PMID]

[2] Arboleda-Flórez J, Sartorius N, editors. Understanding the stigma of mental illness: Theory and interventions. Hoboken, NJ: John Wiley \& Sons, Ltd; 2008. [DOI:10.1002/9780470997642]

[3] Sharifi V, Amin-Esmaeili M, Hajebi A, Motevalian A, Radgoodarzi R, Hefazi M, et al. Twelve-month prevalence and correlates of psychiatric disorders in Iran: The Iranian mental health survey, 2011. Archives of Iranian Medicine. 2015; 18(2):76-84. [PMID]

[4] Azizi F. [Prediction of morbidity and burden of diseases in Iran and world (Persian)]. Research in Medicine. 2008; 32(4):259-60. http:/ / pejouhesh.sbmu.ac.ir/browse.php?a_code=A-10-1$430 \&$ slc_lang $=\mathrm{fa} \& \mathrm{sid}=1$

[5] Karshaki H, Meshkin Yazd A, Soudmand P. [Validation of the mental illness stigma scale, Ibn Sina Hospital, Mashhad (Persian)]. Journal of Mazandaran University of Medical Sciences. 2014; 24(113):73-9. http://jmums.mazums.ac.ir/article1-3794-en.html

[6] Naghavi M, Abolhassani F, Pourmalek F, Jafari N, Moradi Lakeh M, Eshrati B, et al. [The burden of disease and injury in Iran in the year 2003 (Persian)]. Iranian Journal of Epidemiology. 2008; 4(1):1-19. http://irje.tums.ac.ir/article-1-147-en.html

[7] Heydari A, Meshkin Yazd A, Sodmand P. [Stigma in health systems: A review article (Persian)]. Journal of Ilam University of Medical Sciences. 2015; 23(4):217-28. http://sjimu.medilam. ac.ir/article-1-1694-en.html

[8] Gerhardsen G, Hansen AV, Killi M, Fornitz GG, Pedersen F, Roos SB. The efficacy of femal in women with premenstrual syndrome: A randomised, double-blind, parallel-group, placebo-controlled, multicentre study. Advances in Therapy. 2008; 25(6):595. [DOI:10.1007/s12325-008-0072-4] [PMID]

[9] Snyder M. Changing the tide: Stigma, school youth, and mental illness. NASN School Nurse. 2015; 30(2):130-2. [DOI:10.1177/1942602X14553307] [PMID]

[10] Hinshaw SP. The stigmatization of mental illness in children and parents: Developmental issues, family concerns, and research needs. Journal of Child Psychology and Psychiatry. 2005; 46(7):714-34. [DOI:10.1111/j.1469-7610.2005.01456.x] [PMID]

[11] de Sousa S, Marques A, Curral R, Queirós C. Stigmatizing attitudes in relatives of people with schizophrenia: A study using the Attribution Questionnaire AQ-27. Trends in Psychiatry and Psychotherapy. 2012; 34(4):186-97. [DOI:10.1590/S223760892012000400004] [PMID]

[12] Dehkhoda AA. ['Ang (Persian)] [Internet]. 2018 [Updated 2018]. Available from: https://dictionary.abadis.ir/ fatofa/\%D8\%A7\%D9\%86\%DA\%AF

[13] Roohandeh M. [Mark (Persian)] [Internet]. 2015 [Updated 2015]. Available from: http://parsicwords.mihanblog.com/ post/1148

[14] Corrigan P. How stigma interferes with mental health care. American Psychologist. 2004; 59(7):614-25. [DOI:10.1037/0003066X.59.7.614] [PMID]
[15] Kheirabadi GR, Nekouei-Shahraki M, Maracy MR. [Stigma of depression and related factors in university students (Persian)] Journal of Isfahan Medical School. 2017; 35(428):488-95. http:/ / jims.mui.ac.ir/index.php/jims/article/view/7818

[16] Karamlou S, Mottaghipour Y, Borjali A, Sadeghi MS, Khanipour $H$. [Effective factors in experiencing shame in families of patients with severe psychiatric disorders: Perceived stigma, rejection sensitivity, cognitive appraisal (Persian)] Clinical Psychology Studies. 2016; 6(23):25-39. [DOI:10.22054/ JCPS.2016.4556]

[17] Leff J, Warner R. Social inclusion of people with mental illness. Cambridge: Cambridge University Press; 2006. [DOI:10.1017/ CBO9780511543937]

[18] Östman M, Kjellin L. Stigma by association: Psychological factors in relatives of people with mental illness. The British Journal of Psychiatry. 2002; 181(6):494-8. [DOI:10.1192/bjp.181.6.494] [PMID]

[19] Sibitz I, Unger A, Woppmann A, Zidek T, Amering M. Stigma resistance in patients with schizophrenia. Schizophrenia Bulletin. 2011; 37(2):316-23. [DOI:10.1093/schbul/sbp048] [PMID] [PMCID]

[20] Gaebel W, Baumann AE. Interventions to reduce the stigma associated with severe mental illness: Experiences from the open the doors program in Germany. The Canadian Journal of Psychiatry. 2003; 48(10):657-62. [DOI:10.1177/070674370304801003 ] [PMID]

[21] Link BG, Struening EL, Neese-Todd S, Asmussen S, Phelan JC. Stigma as a barrier to recovery: The consequences of stigma for the self-esteem of people with mental illnesses. Psychiatric Services. 2001; 52(12):1621-6. [DOI:10.1176/appi.ps.52.12.1621] [PMID]

[22] Pescosolido BA, Martin JK, Long JS, Medina TR, Phelan JC, Link BG. "A disease like any other"? A decade of change in public reactions to schizophrenia, depression, and alcohol dependence. The American Journal of Psychiatry. 2010; 167(11):1321-30. [DOI:10.1176/appi.ajp.2010.09121743] [PMID] [PMCID]

[23] Sartorius N. Iatrogenic stigma of mental illness. BMJ. 2002 324(7352):1470-1. [DOI:10.1136/bmi.324.7352.1470] [PMID] [PMCID]

[24] Link BG, Cullen FT, Frank J, Wozniak JF. The social rejection of former mental patients: Understanding why labels matter. American Journal of Sociology. 1987; 92(6):1461-500. [DOI:10.1086/228672]

[25] Tavakoli S, Kaviani H, Sharifi V, Sadeghi M, Fotouhi A. [Examining cognitive, emotional and behavioral components of public stigma towards persons with mental illness (Persian)] Advances in Cognitive Sciences. 2006; 8(2):31-43. http://icssjournal.ir/article-1-231-en.html

[26] Tavakoli S, Sharifi V, Taj M, Mohammadi MR. [Stigma of depression and its relationship with attitudes toward seeking professional help among students (Persian)]. Advances in Cognitive Sciences. 2010; 12(3):19-33. http://icssjournal.ir/article1-120-en.htm

[27] Younesi SJ, Akbari-Zardkhaneh S, Behjati Ardakani Z. [Evaluating stigma among infertile men and women in Iran (Persian)] Journal of Reproduction \& Infertility. 2006; 6(5):531-45. https:// www.jri.ir/article/215 
[28] Rezayat F. [Exploring the encounter process with stigma in patients with schizophrenia spectrum disorders and their families and presenting a care model (Persian)] [PhD. dissertation] Tehran: Tarbiat Modares University; 2018. https://parseh. modares.ac.ir $/$ thesis.php?id=3084151\&sid=1\&slc_lang=en

[29] Ranjbar Kermani F, Mazinani R, Fadaei F, Dolatshahi B, Rahgozar M. [Psychometric properties of the Persian version of social distance and dangerousness scales to investigate stigma due to severe mental illness in Iran (Persian)]. Iranian Journal of Psychiatry and Clinical Psychology. 2015; 21(3):254-61. http:/ / ijpcp.iums.ac.ir/article-1-2471-en.html

[30] Rasooli Isini M. [Predicting stigmatizing attitudes toward people with mental illness based on familiarity with the mentally ill in Bandar Abbas students in 2012 (Persian)]. [MSc. thesis]. Shiraz: Shiraz University; 2012. https:/ / www.virascience.com/ thesis/579634

[31] Halter MJ. The stigma of seeking care and depression. Archives of Psychiatric Nursing. 2004; 18(5):178-84. [DOI:10.1016/j. apnu.2004.07.005] [PMID]

[32] Stuart H, Arboleda-Florez J. Community attitudes toward people with schizophrenia. The Canadian Journal of Psychiatry. 2001; 46(3):245-52. [DOI:10.1177/070674370104600304] [PMID]

[33] Nojomi M, Malakouti SK, Ghanean H, Joghataei MT, Jacobson L. [Mental illness stigma in city of Tehran, 2009 (Persian)]. Razi Journal of Medical Sciences. 2010; 17(78-79):45-52. http:/ / rjms.iums.ac.ir/article-1-1606-en.html

[34] Lindsey MA, Joe S, Nebbitt V. Family matters: The role of mental health stigma and social support on depressive symptoms and subsequent help seeking among African American boys. Journal of Black Psychology. 2010; 36(4):458-82. [DOI:10.1177/0095798409355796] [PMID] [PMCID]

[35] Zimmer-Gembeck MJ, Waters AM, Kindermann T. A social relations analysis of liking for and by peers: Associations with gender, depression, peer perception, and worry. Journal of Adolescence. 2010; 33(1):69-81. [DOI:10.1016/j.adolescence.2009.05.005] [PMID]

[36] Szmukler GI, Burgess P, Herrman H, Bloch S, Benson A, Colusa S. Caring for relatives with serious mental illness: The development of the experience of caregiving inventory. Social Psychiatry and Psychiatric Epidemiology. 1996; 31(3-4):137-48. [DOI:10.1007/BF00785760] [PMID]

[37] Motaghipour Y, Shams J, Salesian N, Sharifi V, Alaghband Rad J. [Cultural adaptation, validity and reliability of Persian version of experience of caregiving inventory in families of patients with severe mental disorders (Persian)]. Iranian Journal of Psychiatry and Clinical Psychology. 2011; 17(3):226-31. http:// ijpcp.iums.ac.ir/article-1-1418-en.html

[38] Harvey K, Burns T, Fahy T, Manley C, Tattan T. Relatives of patients with severe psychotic illness: Factors that influence appraisal of caregiving and psychological distress. Social Psychiatry and Psychiatric Epidemiology. 2001; 36(9):456-61. [DOI:10.1007/s001270170024] [PMID]

[39] Addington J, McCleery A, Addington D. Three-year outcome of family work in an early psychosis program. Schizophrenia Research. 2005; 79(1):107-16. [DOI:10.1016/j.schres.2005.03.019]

[40] Fallahi Khesht-Masjedi M, Shokrgozar S, Abdollahi E, Golshahi M, Sharif-Ghaziani Z. Exploring social factors of mental illness stigmatization in adolescents with mental disorders.
Journal of Clinical and Diagnostic Research. 2017; 11(11):VC014. [DOI:10.7860/JCDR/2017/27906.10832]

[41] Rezaee Dehnavi S, Nori A, Jafari M, Faramarzi S. [Investigating stigma phenomenon among mothers with Down syndrome children in Isfahan: A psycho-social approach (Persian)]. Journal of Family Research. 2008; 5(3):401-16. http://jfr.sbu.ac.ir/ article/view/93

[42] Rezaei DehnaviS, Hemati Alamdarloo Gh. [The impact of perceived stigma on mental health of mothers of children with autism spectrum disorders (Persian)]. Journal of Family Research. 2015; 11(1):123-39. http://jfr.sbu.ac.ir/article/view/8358

[43] Kassam A, Papish A, Modgill G, Patten S. The development and psychometric properties of a new scale to measure mental illness related stigma by health care providers: The Opening Minds Scale for Health Care providers (OMS-HC). BMC Psychiatry. 2012; 12:62. [DOI:10.1186/1471-244X-12-62] [PMID] [PMCID]

[44] Kordlou F, Kabir K, Bahador H, Dehghan Nayeri T. [Could nursing educational courses change the attitudes of students to mental disorders stigma? A quasi- experimental study in Alborz University of Medical Sciences (Persian)]. Alborz University Medical Journal. 2015; 4(4):248-54. [DOI:10.18869/acadpub. aums.4.4.248]

[45] Ebrahimi H, Namdar H, Vahidi M. Mental illness stigma among nurses in psychiatric wards of teaching hospitals in the north-west of Iran. Iranian Journal of Nursing and Midwifery Research. 2012; 17(7):534-8. [PMID] [PMCID]

[46] Cohen J, Struening EL. Opinions about mental illness in the personnel of two large mental hospitals. The Journal of Abnormal and Social Psychology. 1962; 64(5):349-60. [DOI:10.1037/ h0045526] [PMID]

[47] Crocetti G, Spiro HR, Siassi I. Are the ranks closed? Attitudinal social distance and mental illness. American Journal of Psychiatry. 1971; 127(9):1121-7. [DOI:10.1176/ajp.127.9.1121] [PMID]

[48] Ghasemi A, Fatemi MM. [The effect of an educational film on the attitudes of mentally ill patients' families toward mental illness (Persian)]. [MD. Dissertation]. Tehran: Tehran University of Medical Sciences 1999.

[49] Kheirabadi G, Yousefi F, Vafaei F. [Attitude toward mental illness in Sanandaj (Persian)]. Scientific Journal of Kurdistan University of Medical Sciences. 1997; 2:14-7

[50] Amini H, Majdzadeh R, Eftekhar-Ardebili H, Shabani A, Davari-Ashtiani R. How mental illness is perceived by Iranian medical students: A preliminary study. Clinical Practice \& Epidemiology in Mental Health. 2013; 9:62-8. [DOI:10.2174/174501 7901309010062] [PMID] [PMCID]

[51] Amini H, Shoar S, Tabatabaee M, Arabzadeh S. The effect of clinical exposure to patients on medical students' attitude towards mental illness. Iranian Journal of Psychiatry and Behavioral Sciences. 2016; 10(3):e1887. [DOI:10.17795/ijpbs-1887] [PMID] [PMCID]

[52] Griffiths KM, Christensen H, Jorm AF, Evans K, Groves C. Effect of web-based depression literacy and cognitive-behavioural therapy interventions on stigmatising attitudes to depression: Randomised controlled trial. The British Journal of Psychiatry. 2004; 185(4):342-9. [DOI:10.1192/bjp.185.4.342] [PMID] 
[53] Griffiths KM, Christensen H, Jorm AF. Predictors of depression stigma. BMC Psychiatry. 2008; 8:25. [DOI:10.1186/1471244X-8-25] [PMID] [PMCID]

[54] King M, Dinos S, Shaw J, Watson R, Stevens S, Passetti F, et al. The stigma scale: Development of a standardised measure of the stigma of mental illness. The British Journal of Psychiatry. 2007; 190(3):248-54. [DOI:10.1192/bjp.bp.106.024638] [PMID]

[55] Sohrabi F, Esfandyari G, Yousefi F, Abdollahi N, Saed G, Bakhivushi Sh. [The relationships between self-esteem, demographic variables, psychiatric diagnosis and frequency of hospitalization with mental illness stigma in psychiatric patients (Persian)]. Shenakht Journal of Psychology and Psychiatry. 2016; 3(4):27-38. http://shenakht.muk.ac.ir/article-1-268-en.html

[56] Ritsher JB, Otilingam PG, Grajales M. Internalized stigma of mental illness: Psychometric properties of a new measure. Psychiatry Research. 2003; 121(1):31-49. [DOI:10.1016/j.psychres.2003.08.008] [PMID]

[57] Zisman-Ilani Y, Levy-Frank I, Hasson-Ohayon I, Kravetz Sh, Mashiach-Eizenberg M, Roe D. Measuring the internalized stigma of parents of persons with a serious mental illness: The factor structure of the parents' internalized stigma of mental illness scale. The Journal of Nervous and Mental Disease. 2013; 201(3):183-7. [DOI:10.1097/NMD.0b013e3182845d00] [PMID]

[58] Ghanean H, Nojomi M, Jacobsson L. Internalized stigma of mental illness in Tehran, Iran. Stigma Research and Action. 2011; 1(1):11-7. [DOI:10.5463/sra.v1i1.10]

[59] Vaghee S, Salarhaji A, Asgharipour N, Chamanzari H. [Effects of psychoeducation on stigma in family caregivers of patients with schizophrenia: A clinical trial (Persian)]. Evidence Based Care Journal. 2015; 5(3):63-76. [DOI:10.22038/EBCJ.2015.5159]

[60] Girma E, Möller-Leimkühler AM, Dehning S, Mueller N, Tesfaye M, Froeschl G. Self-stigma among caregivers of people with mental illness: Toward caregivers' empowerment. Journal of Multidisciplinary Healthcare. 2014; 7:37-43. [DOI:10.2147/JMDH. S57259] [PMID] [PMCID]

[61] Gonzalez JM, Perlick DA, Miklowitz DJ, Kaczynski R, Hernandez M, Rosenheck RA, et al. Factors associated with stigma among caregivers of patients with bipolar disorder in the STEPBD study. Psychiatric Services. 2007; 58(1):41-8. [DOI:10.1176/ ps.2007.58.1.41] [PMID]

[62] London C. Stigma and mental illness: A comparative study of attitudes and personal constructs [PhD. dissertation]. London: Brunel University; 2010. https://bura.brunel.ac.uk/handle/2438/4447

[63] Shamsaei F, Sadeghian E, Nazari F, Barzegar A. [Considering Stigma in Family Caregivers of Patients with Psychiatric Disorders in Farshchian Hospital, Hamadan, Iran (Persian)]. Journal of Health System Research. 2018; 13(4):422-8. http:/ / hsr.mui.ac.ir/ article-1-983-en.html

[64] Vaghee S, Kashani Lotfabadi M, Salarhaji A, Vaghei N, Hashemi BM. Comparing the effects of contact-based education and acceptance and commitment-based training on empathy toward mental illnesses among nursing students. Iranian Journal of Psychiatry. 2018; 13(2):119-27. [PMID] [PMCID]

[65] Sadeghi M, Kaviani H, Rezai R. [Stigma of mental disorder among families of patients with major depressive disorder, bipolar disorder and schizophrenia (Persian)]. Advances in Cognitive Science. 2003; 5(2):16-25. http://icssjournal.ir/article-1-163-en. html
[66] Rüsch N, Corrigan PW, Powell K, Rajah A, Olschewski M, Wilkniss $\mathrm{S}$, et al. A stress-coping model of mental illness stigma: II. Emotional stress responses, coping behavior and outcome. Schizophrenia Research. 2009; 110(1-3):65-71. [DOI:10.1016/j. schres.2009.01.005] [PMID] [PMCID]

[67] Berenson KR, Gyurak A, Ayduk Ö, Downey G, Garner MJ, Mogg K, et al. Rejection sensitivity and disruption of attention by social threat cues. Journal of Research in Personality. 2009; 43(6):1064-72. [DOI:10.1016/j.jrp.2009.07.007] [PMID] [PMCID]

[68] Downey G, Feldman SI. Implications of rejection sensitivity for intimate relationships. Journal of Personality and Social Psychology. 1996; 70(6):1327-43. [DOI:10.1037/0022-3514.70.6.1327] [PMID]

[69] Hosseini Tari ZS. [Stress, self-efficacy, parenting guilt feeling perceived stigma amongst mothers of Attention Deficient Hyperactivity Disorder patients, considering demographic factors (Persian)]. Paper presented at: $5^{\text {th }}$ National Conference on Recent Innovations in Psychotherapy Application and Empowerment; 21 June 2019; Tehran, Iran. https:/ / civilica.com/doc/922447/

[70] Link BG, Yang LH, Phelan JC, Collins PY. Measuring mental illness stigma. Schizophrenia Bulletin. 2004; 30(3):511-41. [DOI:10.1093/oxfordjournals.schbul.a007098] [PMID]

[71] Corrigan P, Markowitz FE, Watson A, Rowan D, Kubiak MA. An attribution model of public discrimination towards persons with mental illness. Journal of Health and Social Behavior. 2003; 44(2):162-79. [DOI:10.2307/1519806] [PMID]

[72] Corrigan PW, Green A, Lundin R, Kubiak MA, Penn DL. Familiarity with and social distance from people who have serious mental illness. Psychiatric Services . 2001; 52(7):953-8. [DOI:10.1176/appi.ps.52.7.953] [PMID]

[73] Holmes EP, Corrigan PW, Williams P, Canar J, Kubiak MA Changing attitudes about schizophrenia. Schizophrenia Bulletin. 1999; 25(3):447-56. [DOI:10.1093/oxfordjournals.schbul.a033392] [PMID]

[74] Fischer EH, Farina A. Attitudes toward seeking professional psychologial help: A shortened form and considerations for research. Journal of College Student Development. 1995; 36(4):36873. [DOI:10.1037/t05375-000] 
This Page Intentionally Left Blank 